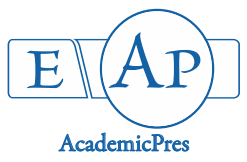

Pattanaik N et al. (2021)

Notulae Scientia Biologicae

Volume 13, Issue 3, Article number 10934

DOI: $10.15835 /$ nsb13310934

Research Article

\title{
Moth (Lepidoptera: Heterocera) diversity of Bhubaneswar, Odisha, India: a preliminary study
}

\author{
Nivedita PATTANAIK ${ }^{1}$, Aryjit SATAPATHY ${ }^{1}$, \\ Devi PRIYADARSHINI ${ }^{2 *}$
}

\author{
${ }^{1}$ Buxi Jagabandhu Bidyadhar Autonomous College, BJB Nagar, Bhubaneswar 751014, Odisha, \\ India;nivedita.p22@gmail.com; aryjit89@gmail.com \\ ${ }^{2}$ Regional Museum of Natural History, PO-RRL, Acharya Vihar, Bhubaneswar 751013, Odisha, \\ India; deviwildlife@gmail.com (" ${ }^{*}$ corresponding author)
}

\begin{abstract}
A preliminary checklist has been compiled to study the moth diversity of Bhubaneswar, Odisha, an eastern state of India. The present study has recorded a total of 154 species belonging to 129 genera and 19 families. The highest diversity of moths was recorded in the family Crambidae ( 48 species, 38 genera), followed by the families Erebidae ( 42 species, 37 genera), Geometridae ( 15 species, 12 genera), Noctuidae (13 species, 11 genera) and others. The study was conducted over a period of 18 months from May 2019 to October 2020. Here we present an illustrated checklist of 154 moth species from Bhubaneswar which improves our insight into the lesser-known lepidopterans from the state of Odisha. This shall further help us strengthen our knowledge about the importance of moths in our environment and contribute towards its conservation at large.

Keywords: checklist; conservation; documentation; Khordha; moth species; urban habitat

\section{Introduction}

Moths are biologically, economically (Sharma and Bisen, 2013) and aesthetically a very important group of insects (Devoto et al., 2011; Le Croy et al., 2013; Dey et al., 2015). They are one of the most heterogeneous groups of insects (Soggard, 2009) consisting of around 1,27,000 species identified around the world as estimated by Hamlyn in 1969 (Alfred et al., 1998) and around 12,000 species reported from India alone (Chandra and Nema, 2007).

India lies in the Indo-Malayan biogeographic realm of the world and is listed amongst the 17 mega biodiverse countries. It consists of four biodiversity hotspots which indicates the uniqueness of its flora and fauna. It shelters around $6.5 \%$ of the species known across the globe on $2.4 \%$ of the world's total area (Faunal Diversity of India, 2020; http://www.zsienvis.nic.in/).

Odisha is unique in its geographic location with major part of the state falling in the Deccan Peninsula including Chhota Nagpur Province and Eastern Highlands while it is guarded by a $480 \mathrm{kms}$ long coastline on its east. Since a considerable part of the Eastern Ghats falls within the territory of Odisha, it is speculated that the diversity of moths will be unique and interesting to investigate. In Odisha, the earliest works on moths have
\end{abstract}

Received: 24 Mar 2021. Received in revised form: 13 Aug 2021. Accepted: 22 Aug 2021. Published online: 02 Sep 2021.

From Volume 13, Issue 1, 2021, Notulae Scientia Biologicae journal uses article numbers in place of the traditional method of continuous pagination through the volume. The journal will continue to appear quarterly, as before, with four annual numbers. 
been contributed by Hampson $(1892,1894,1895,1896)$ in the Fauna of British India. The State Fauna of Odisha (Part-III) by ZSI (Mandal and Maulik, 1991) reported 87 species under 3 families. There have been several records of moths as pest insects from various studies done in the crop fields. Of these some prominent works are those of paddy (Arora, 2000; Rath et al., 2020), brinjal (Kar et al., 2020), tomato (Sridhar and Srinivas, 2019) and teak (Tripathy et al., 2018); but no compiled work on the diversity of moths has yet been done in the present study area from the capital city of Odisha. However, in a recent work, Jena et al. (2018) reported 30 species from Gupteswar of Koraput district. In the present study, we have investigated the moth diversity primarily of Bhubaneswar city and adjoining urban areas under Khordha district, Odisha, India. A preliminary checklist containing 154 species under 19 families is presented here from the survey of ten study sites over a period of 18 months from May 2019 to October 2020.

\section{Materials and Methods}

The biodiversity documentation of moths has been primarily done in the urban areas of Bhubaneswar $\left(20.2961^{\circ} \mathrm{N}, 85.8245^{\circ} \mathrm{E}\right.$ ), and its outskirts from May 2019 to October 2020 (Figure 1). The state lies in the tropical region and experiences a tropical savanna climate. It witnesses an average annual rainfall of about 1451.2mm (Envis Centre of Odisha, 2020; http://www.orienvis.nic.in/). The district of Khordha has mostly open forests with some moderately dense forests and scrub vegetation. Bhubaneswar is enveloped on one side by Chandaka with semi-evergreen forests and surrounded mostly by dry deciduous forests on its other boundaries. The selected sites for the study were namely, Acharya Vihar (S1), Jaydev Vihar (S2), BJB Nagar (S3), Saheed Nagar (S4), Khandagiri (S5), Pokhariput (S6), Ghangapatna (S7), Dhauli (S8), Dalua (S9) and Raghunathpur (S10) as detailed with GPS locations in Table 1, Figure 2 . The regions prominently have urban habitat with fragmented vegetation. Khordha district has a geographical area of $2813 \mathrm{sq}$. km. of which $456 \mathrm{sq}$. $\mathrm{km}$. has forest cover (Envis Centre of Odisha, 2020; http://www.orienvis.nic.in/).

Table 1. Coordinates of study sites in Bhubaneswar

\begin{tabular}{|c|c|c|}
\hline $\begin{array}{c}\text { Study } \\
\text { site }\end{array}$ & $\begin{array}{c}\text { Name of the study } \\
\text { site }\end{array}$ & Coordinates \\
\hline S1 & Acharya Vihar & $\begin{array}{c}20.2994^{\circ} \mathrm{N}, \\
85.8319^{\circ} \mathrm{E}\end{array}$ \\
\hline S2 & Jaydev Vihar & $\begin{array}{c}20.2997^{\circ} \mathrm{N} \\
85.8173^{\circ} \mathrm{E}\end{array}$ \\
\hline S3 & BJB Nagar & $\begin{array}{c}20.2506^{\circ} \mathrm{N}, \\
85.8448^{\circ} \mathrm{E}\end{array}$ \\
\hline S4 & Saheed Nagar & $\begin{array}{l}20.2910^{\circ} \mathrm{N} \\
85.8456^{\circ} \mathrm{E}\end{array}$ \\
\hline S5 & Khandagiri & $\begin{array}{l}20.2569^{\circ} \mathrm{N} \\
85.7792^{\circ} \mathrm{E}\end{array}$ \\
\hline S6 & Pokhariput & $\begin{array}{c}20.2408^{\circ} \mathrm{N} \\
85.8064^{\circ} \mathrm{E}\end{array}$ \\
\hline S7 & Ghangapatna & $\begin{array}{c}20.3088^{\circ} \mathrm{N}, \\
85.7308^{\circ} \mathrm{E}\end{array}$ \\
\hline S8 & Dhauli & $\begin{array}{c}20.1882^{\circ} \mathrm{N}, \\
85.8448^{\circ} \mathrm{E}\end{array}$ \\
\hline S9 & Dalua & $\begin{array}{c}20.3634^{\circ} \mathrm{N}, \\
85.7176^{\circ} \mathrm{E} \\
\end{array}$ \\
\hline S10 & Raghunathpur & $\begin{array}{c}20.3782^{\circ} \mathrm{N}, \\
85.8278^{\circ} \mathrm{E}\end{array}$ \\
\hline
\end{tabular}



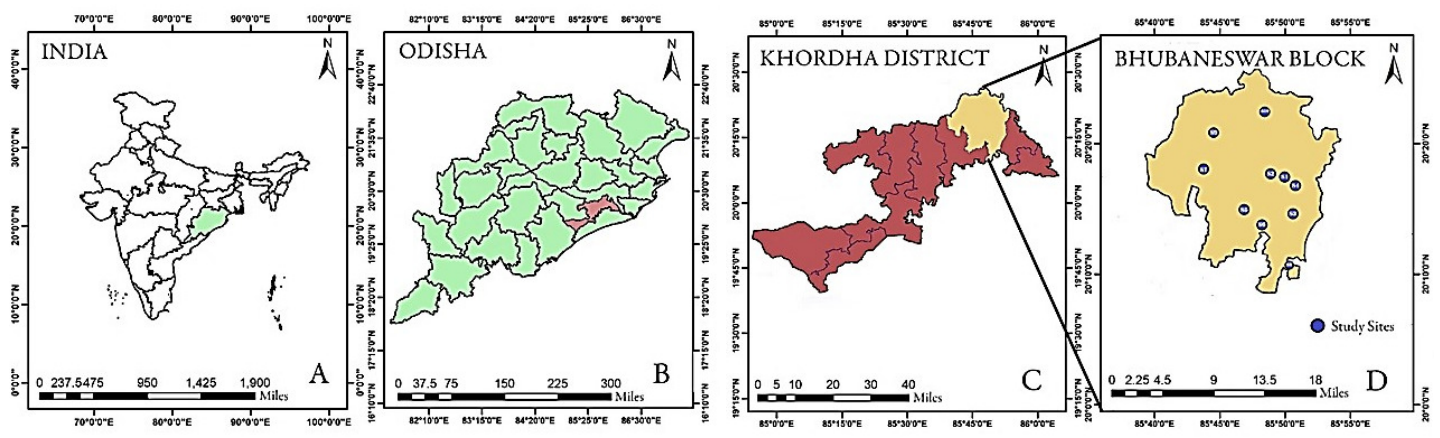

Figure 1. Map of Study Area: A. India, B. Odisha State, C. Khordha District, D. Bhubaneswar Block with site locations

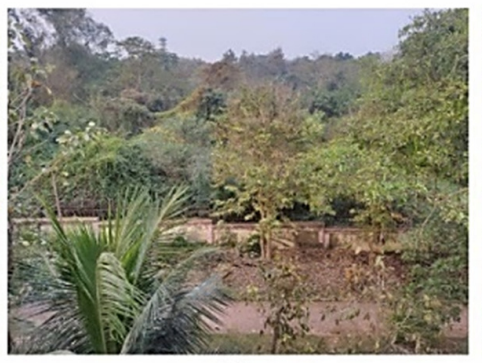

S1

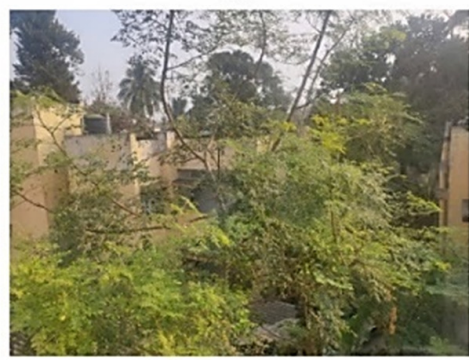

S4

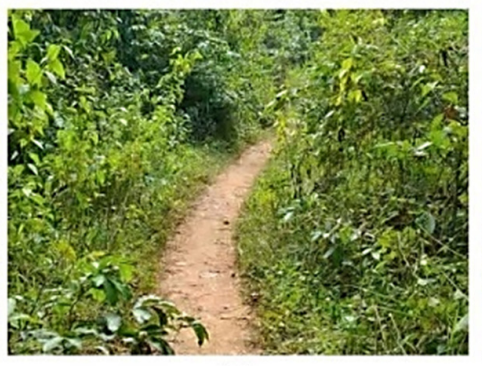

S7

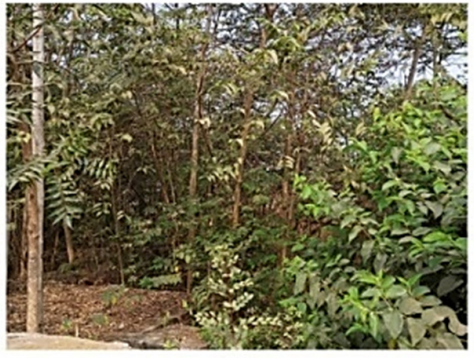

S2

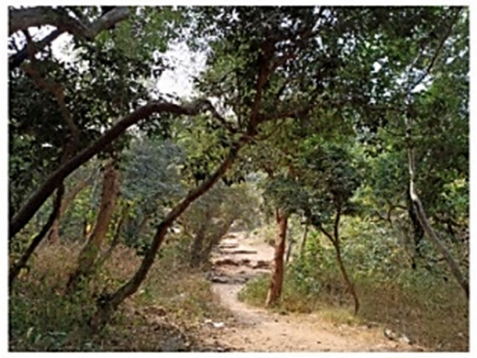

S5

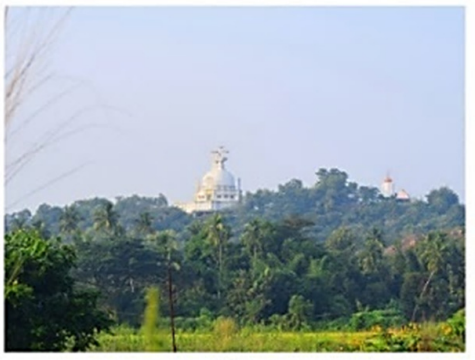

S8

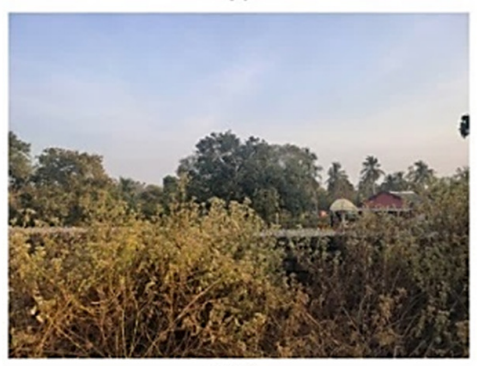

$\mathrm{S} 10$

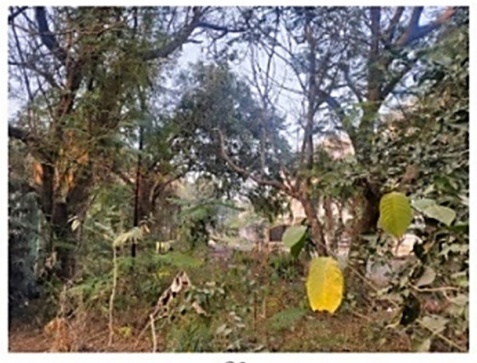

S3

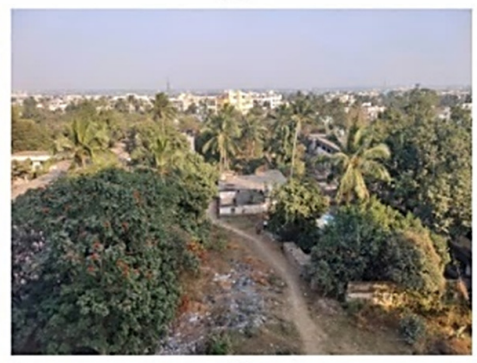

S6

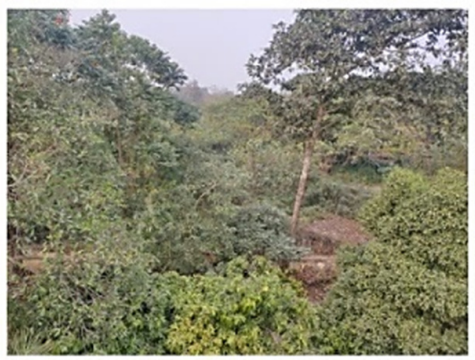

S9

Figure 2. Study sites photographs (S1-S10) 
The moths have been found by random sampling, opportunistic sightings and by setting up of light traps in some of the mentioned locations. The study areas have been searched extensively in the morning $(6: 00 \mathrm{hrs}-$ 8:00hrs) and evening (16:00hrs-19:00 hrs). Net sweeping was done with a standard-sized butterfly net for the day-flying moths and during the evenings for suitable photography from closer angles. Each study site was visited for around 20 days in every season. The light traps had been set in selective study sites using 100-Watt bulbs, which were placed in front of a $15 \mathrm{ft} \times 5 \mathrm{ft}$ white cloth supported by the wall, for about 15 nights in every season (Figure 3). Standard tungsten bulbs were used for moth trapping. Efforts were made to create the least disturbance for the creatures in their natural environment while resting, feeding etc. except for instances when it was required to be caught for photography.

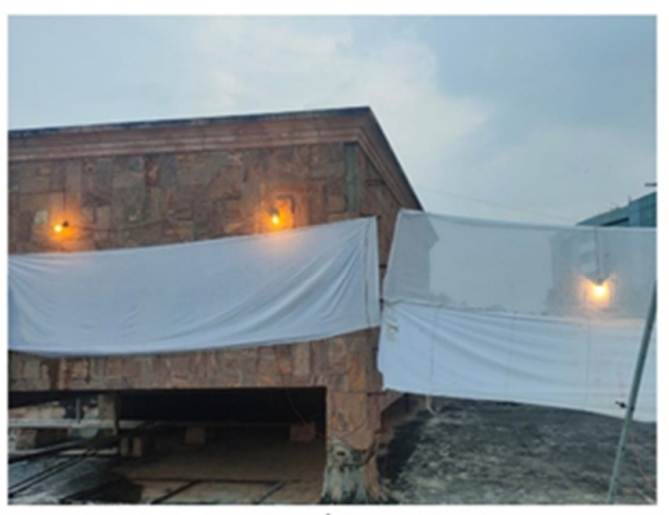

A

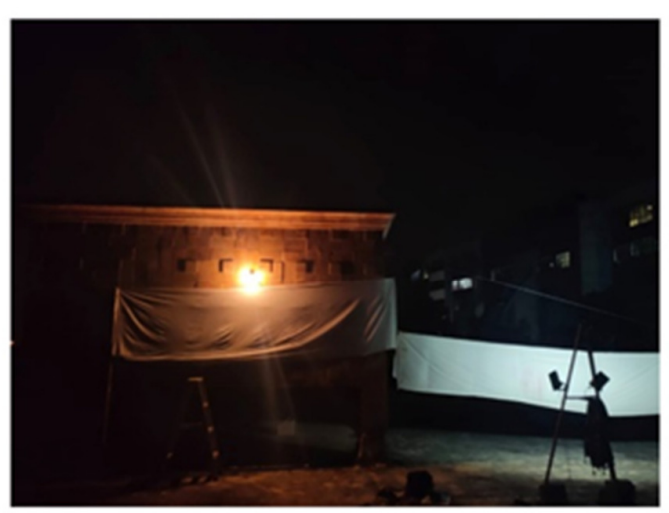

$\mathrm{B}$

Figure 3. Moth light trap: A. During evening; B. During night

Moths were photographed using DSLR cameras (Nikon D5300, 18-55mm and 70-300mm lens; and Canon EOS 80D, Tamron 90mm lens) and smartphone cameras. Identification was done by referring to the available literature (Hampson, 1892-1896; Bell and Scott, 1937; Holloway, 1985-2011; Shubhalaxmi et al., 2011; Kononenko and Pinratana, 2013; Dey et al., 2018). Some online sources like Moths of India database (Sondhi et al. 2021; http://www.mothsofindia.org); India Biodiversity Portal database (Vattakaven et al., 2016; https://indiabiodiversity.org), Natural History Museum database (HOSTS, 2020; https://www.nhm.ac.uk/), National Bureau of Agricultural Insect Resources database (Insect Pests, 2020; https://databases.nbair.res.in/) and iNaturalist database (iNaturalist, 2020; https://www.inaturalist.org/), were quite helpful in the process of identification apart from the published references. Museum collections in Lepidoptera section from Regional Museum of Natural History, Bhubaneswar were also referred for identification of some of the macrolepidoptera moths. For the present study, none of the moths was collected or killed and therefore live photography of the moths was done as presented in the image plates. Due to several constraints, the identification was primarily done based on external morphological characters and no sophisticated methods such as genitalia dissection, DNA barcoding etc. were used to identify the moth species.

The system of classification detailed by Van Nieukerken et al. (2011) has been followed for identifying moths to the families. This method mostly follows the classification by Kristensen (1999), Kristensen et al. (2007) as well as the recent developments by Zahiri et al. (2010,2011). A few of the moths have been assigned only to the genus as the morphological identification was not enough for many individuals to designate them to species level. There have been repetitive observations of the same moth species in different survey sites. In such cases, only one observation has been taken into consideration. The map has been created in ArcGIS, using

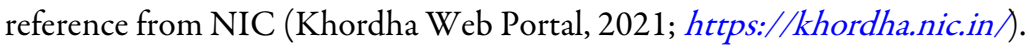




\section{Results and Discussion}

We examined major studies on moths from the eastern region of India in the post-Victorian era. Saha and Raychaudhuri (1998) reported about 31 moths from West Bengal while Gurule and Nikam (2013) reported that Ghosh in 2003 documented 260 moths only in the family Geometridae from the same state. Further, Sanyal et al. (2012) also compiled 707 moths from West Bengal. Chandra and Nema (2007) reported 142 moths from Madhya Pradesh and Chhattisgarh, Singh and Ranjan (2016) added 23 new species from the superfamily Noctuoidea to the list of 138 moths from Dalma wildlife sanctuary. Singh et al. (2018) have reported 140 species of moths from Koderma, Jharkhand. From the information available about the moth fauna of Odisha state, it is understood that scanty studies have been done and few species reported till date about non-pest moths from the state. Studies done by (Mandal and Maulik, 1991) reports 87 species of moths in the Fauna of Orissa (Part-III) by ZSI out of which only six moth species were found in the present study. Seven moths found in this study were also reported by Jena et al. (2018). Although the moth Glyphodes bicolor has been reported by Jena et al. (2018), it appears to be a case of misidentification, which as per the pictures provided in the paper, suggest the same to be Glyphodes bivitralis Guenée, 1854. This was identified from various online resources like Moths of India database (Sondhi et al., 2021; http://www.mothsofindia.org/), iNaturalist database (iNaturalist, 2020; https://www.inaturalist.org/) and confirmed from other available literature.

Although there have been some scattered works on the pest moths of various crops from the state, the present study is an attempt to come up with a compiled checklist to enlist the diversity of moth fauna from Bhubaneswar. In the present study, a total of 154 moths have been identified out of the several individuals recorded, belonging to 19 families and 12 superfamilies from surveys in ten different study sites across Bhubaneswar city and its outskirts as presented in Table 2, Plates 1- 5. All the photographs have been contributed by the authors unless credited otherwise.

Table 2. Preliminary checklist of moths recorded during the study at various study sites

\begin{tabular}{|c|c|c|c|c|c|}
\hline $\begin{array}{l}\text { Sl. } \\
\text { No. }\end{array}$ & Subfamily & Scientific name & Common name & $\begin{array}{l}\text { Author and year } \\
\text { of description }\end{array}$ & Survey site \\
\hline \multicolumn{6}{|c|}{ Superfamily Tineoidea } \\
\hline \multicolumn{6}{|c|}{ Family Tineidae } \\
\hline 1 & Acrolophinae & Acrolophus sp. & & & S1 \\
\hline \multicolumn{6}{|c|}{ Superfamily Yponomeutoidea } \\
\hline \multicolumn{6}{|c|}{ Family Attevidae } \\
\hline 2 & Attevinae & Attevasp. & & & S3 \\
\hline \multicolumn{6}{|c|}{ Superfamily Gelechioidea } \\
\hline \multicolumn{6}{|c|}{ Family Lecithoceridae } \\
\hline 3 & Lecithocerinae & Lecithocera sp. & & & S2 \\
\hline \multicolumn{6}{|c|}{ Family Scythrididae } \\
\hline 4 & Scythridinae & Eretmocera impactella & & (Walker, 1864) & S1 \\
\hline \multicolumn{6}{|c|}{ Superfamily Tortricoidea } \\
\hline \multicolumn{6}{|c|}{ Family Tortricidae } \\
\hline 5 & Tortricinae & Adoxophyes fasciculana & & (Walker, 1866) & S4 \\
\hline 6 & Tortricinae & Archips micaceana & & (Walker, 1863) & S4 \\
\hline \multicolumn{6}{|c|}{ Superfamily Zygaenoidea } \\
\hline \multicolumn{6}{|c|}{ Family Limacodidae } \\
\hline 7 & Limacodinae & Aphendala tripartita & & Moore, 1884 & S3 \\
\hline 8 & Limacodinae & Parasa sp. & & & S2 \\
\hline \multicolumn{6}{|c|}{ Superfamily Thyridoidea } \\
\hline
\end{tabular}


Pattanaik N et al. (2021). Not Sci Biol 13(3):10934

\begin{tabular}{|c|c|c|c|c|c|}
\hline \multicolumn{6}{|c|}{ Family Thyrididae } \\
\hline 9 & Striglininae & Banisia sp. & & & S3 \\
\hline \multicolumn{6}{|c|}{ Superfamily Hyblaeoidea } \\
\hline \multicolumn{6}{|c|}{ Famliy Hyblaeidae } \\
\hline 10 & & Hyblaea sp. & & & S3 \\
\hline \multicolumn{6}{|c|}{ Superfamily Pyraloidea } \\
\hline \multicolumn{6}{|c|}{ Family Pyralidae } \\
\hline 11 & Pyralinae & Endotricha mesenterialis & & (Walker, 1859) & S3 \\
\hline 12 & Pyralinae & Endotricha repandalis & & Fabricius, 1794 & S4 \\
\hline 13 & Pyralinae & Hypsopygia sp. & & & S3 \\
\hline 14 & Pyralinae & Pyralis manihotalis & $\begin{array}{c}\text { Tropical Meal } \\
\text { Moth }\end{array}$ & Guenée, 1854 & S3 \\
\hline 15 & Pyralinae & Pyralis pictalis & $\begin{array}{c}\text { Painted Meal } \\
\text { Moth } \\
\end{array}$ & (Curtis, 1834) & S3 \\
\hline 16 & Pyralinae & Sacada sp. & & & S2 \\
\hline 17 & Pyralinae & Zitha torridalis & & (Lederer, 1863) & S8 \\
\hline \multicolumn{6}{|c|}{ Family Crambidae } \\
\hline 18 & Acentropinae & Parapoynx fluctuosalis & & (Meyrick, 1899) & S6 \\
\hline 19 & Acentropinae & Parapoynx stagnalis & & (Zeller, 1852) & S3 \\
\hline 20 & Crambinae & Ancylolomia sp. & & & S3 \\
\hline 21 & Glaphyriinae & Noorda blitealis & & Walker, 1859 & S3 \\
\hline 22 & Pyraustinae & Isocentris filalis & & (Guenée, 1854) & S1 \\
\hline 23 & Pyraustinae & Pagyda salvalis & & Walker, 1859 & S3 \\
\hline 24 & Pyraustinae & Paliga sp. & & & S1 \\
\hline 25 & Schoebiinae & Scirpophaga incertulas & $\begin{array}{l}\text { Yellow Stem } \\
\text { Borer Moth }\end{array}$ & (Walker, 1863) & S3 \\
\hline 26 & Schoebiinae & Scirpophaga nivella & & (Fabricius, 1794) & S3 \\
\hline 27 & Spilomelinae & Arthroschista hilaralis & & (Walker, 1859) & S3 \\
\hline 28 & Spilomelinae & Bradina admixtalis & & (Walker, 1859) & S4 \\
\hline 29 & Spilomelinae & Chalcidoptera appensalis & & Snellen, [1884] & S3 \\
\hline 30 & Spilomelinae & Cnaphalocrocis medinalis & & (Guenée, 1854) & S3 \\
\hline 31 & Spilomelinae & Cnaphalocrocis ruralis & & (Walker, 1859) & S3 \\
\hline 32 & Spilomelinae & Conogethes sp. & & & S1 \\
\hline 33 & Spilomelinae & Cydalima laticostalis & & (Guenée, 1854) & $\mathrm{S} 10$ \\
\hline 34 & Spilomelinae & Diaphania indica & $\begin{array}{c}\text { Cucumber } \\
\text { Moth }\end{array}$ & (Saunders, 1851) & S1 \\
\hline 35 & Spilomelinae & Dysallacta negatalis & $\begin{array}{l}\text { Karanj Leaf } \\
\text { Borer Moth }\end{array}$ & (Walker, 1859) & S1 \\
\hline 36 & Spilomelinae & Elophila difflualis & & (Snellen, 1880) & S3 \\
\hline 37 & Spilomelinae & Endocrossis flavibasalis & & (Moore, 1867) & S8 \\
\hline 38 & Spilomelinae & Eurrhyparodes tricoloralis & & (Zeller, 1852) & S1 \\
\hline 39 & Spilomelinae & Glyphodes actorionalis & & Walker, 1859 & S1 \\
\hline 40 & Spilomelinae & Glyphodes bicolor & & (Swainson, 1821) & S3 \\
\hline
\end{tabular}


Pattanaik N et al. (2021). Not Sci Biol 13(3):10934

\begin{tabular}{|c|c|c|c|c|c|}
\hline 41 & Spilomelinae & Glyphodes caesalis & $\begin{array}{c}\text { Jack Fruit Borer } \\
\text { Moth }\end{array}$ & (Walker, 1859) & S3 \\
\hline 42 & Spilomelinae & Glyphodes canthusalis & & Walker, 1859 & S2 \\
\hline 43 & Spilomelinae & Haritalodes derogata & & (Fabricius, 1775) & S2 \\
\hline 44 & Spilomelinae & Herpetogramma basalis & & (Walker, 1866) & S3 \\
\hline 45 & Spilomelinae & $\begin{array}{c}\text { Herpetogramma } \\
\text { phaeopteralis }\end{array}$ & $\begin{array}{c}\text { Tropical Sod } \\
\text { Webworm } \\
\text { Moth }\end{array}$ & (Guenée, 1854) & S7 \\
\hline 46 & Spilomelinae & Herpetogramma rudis & & (Warren, 1892) & S3 \\
\hline 47 & Spilomelinae & Hydriris ornatalis & $\begin{array}{c}\text { Ornate Hydriris } \\
\text { Moth }\end{array}$ & $\begin{array}{c}\text { (Duponchel, } \\
1832)\end{array}$ & S3 \\
\hline 48 & Spilomelinae & Leucinodes orbonalis & $\begin{array}{c}\text { Eggplant Fruit } \\
\text { and Shoot Borer } \\
\text { Moth }\end{array}$ & Guenée, 1854 & S3 \\
\hline 49 & Spilomelinae & Mabra eryxalis & & (Walker, 1859) & S2 \\
\hline 50 & Spilomelinae & Maruca vitrata & $\begin{array}{l}\text { Maruca Pod } \\
\text { Borer Moth }\end{array}$ & (Fabricius, 1787) & S6 \\
\hline 51 & Spilomelinae & Metoeca foedalis & & (Guenée, 1854) & S1 \\
\hline 52 & Spilomelinae & Nausinoe geometralis & & (Guenée, 1854) & S8 \\
\hline 53 & Spilomelinae & Nausinoe perspectata & & (Fabricius, 1775) & S8 \\
\hline 54 & Spilomelinae & Nosophora sp. & & & S2 \\
\hline 55 & Spilomelinae & Omiodes indicata & & (Fabricius, 1775) & S5 \\
\hline 56 & Spilomelinae & Pachynoasp. & & & S8 \\
\hline 57 & Spilomelinae & Parotis sp. & & & S3 \\
\hline 58 & Spilomelinae & Pycnarmon cribrata & $\begin{array}{c}\text { Leaf Folder } \\
\text { Moth } \\
\end{array}$ & (Fabricius, 1794) & S1 \\
\hline 59 & Spilomelinae & Pycnarmon virgatalis & & Moore, 1867 & $\mathrm{~S} 2$ \\
\hline 60 & Spilomelinae & Pygospila tyres & $\begin{array}{c}\text { Spotted Grass } \\
\text { Moth }\end{array}$ & (Cramer, [1780]) & S3 \\
\hline 61 & Spilomelinae & Sameodes cancellalis & & (Zeller, 1852) & S1 \\
\hline 62 & Spilomelinae & Spoladea recurvalis & $\begin{array}{c}\text { Beet Webworm } \\
\text { Moth }\end{array}$ & (Fabricius, 1775) & S3 \\
\hline 63 & Spilomelinae & Synclera traducalis & & (Zeller, 1852) & S1 \\
\hline 64 & Spilomelinae & Syngamia latimarginalis & & (Walker, 1859) & S1 \\
\hline 65 & Spilomelinae & Tatobotys biannulalis & & (Walker, 1866) & S3 \\
\hline \multicolumn{6}{|c|}{ Superfamily Lasiocampoidea } \\
\hline \multicolumn{6}{|c|}{ Family Lasiocampidae } \\
\hline 66 & Lasiocampinae & Trabala vishnou & $\begin{array}{l}\text { Rose Myrtle } \\
\text { Lappet Moth }\end{array}$ & (Lefèbvre, 1827) & S1 \\
\hline \multicolumn{6}{|c|}{ Superfamily Bombycoidea } \\
\hline \multicolumn{6}{|c|}{ Family Eupterotidae } \\
\hline 67 & Eupterotinae & Eupterote bifasciata & & Kishida, 1994 & S3 \\
\hline 68 & Eupterotinae & Eupterote undata & & $\begin{array}{c}\text { Blanchard, } \\
{[1844]}\end{array}$ & S3 \\
\hline \multicolumn{6}{|c|}{ Family Bombycidae } \\
\hline 69 & Bombycinae & Trilocha varians & & (Walker, 1855) & S3 \\
\hline
\end{tabular}


Pattanaik N et al. (2021). Not Sci Biol 13(3):10934

\begin{tabular}{|c|c|c|c|c|c|}
\hline \multicolumn{6}{|c|}{ Family Sphingidae } \\
\hline 70 & Macroglossinae & Daphnis nerii & $\begin{array}{l}\text { Oleander } \\
\text { Hawkmoth }\end{array}$ & (Linnaeus, 1758) & S3 \\
\hline 71 & Macroglossinae & Hippotion celerio & $\begin{array}{c}\text { Silver-Striped } \\
\text { Hawkmoth }\end{array}$ & (Linnaeus, 1758) & S3 \\
\hline 72 & Macroglossinae & Macroglossum sp. & & & S1 \\
\hline 73 & Macroglossinae & Theretra lucasii & $\begin{array}{c}\text { Lucas's } \\
\text { Hawkmoth }\end{array}$ & (Walker, 1856) & S1 \\
\hline 74 & Macroglossinae & Theretra oldenlandiae & $\begin{array}{c}\text { White-Banded } \\
\text { Hunter } \\
\text { Hawkmoth }\end{array}$ & (Fabricius, 1775) & S1 \\
\hline 75 & Sphinginae & Acherontia styx & $\begin{array}{c}\text { Lesser Death's } \\
\text { Head } \\
\text { Hawkmoth } \\
\end{array}$ & $\begin{array}{c}\text { (Westwood, } \\
1847 \text { ) }\end{array}$ & S4 \\
\hline 76 & Sphinginae & Psilogramma sp. & & & S1 \\
\hline \multicolumn{6}{|c|}{ Superfamily Geometroidea } \\
\hline \multicolumn{6}{|c|}{ Family Uraniidae } \\
\hline 77 & Epipleminae & Phazaca theclata & $\begin{array}{l}\text { Cotton Leaf } \\
\text { Roller Moth }\end{array}$ & (Guenée, 1857) & S3 \\
\hline 78 & Microniinae & Micronia aculeata & & Guenée, 1857 & S7 \\
\hline \multicolumn{6}{|c|}{ Family Geometridae } \\
\hline 79 & Desmobathrinae & Eumelea ludovicata & & Guenée, [1858] & S4 \\
\hline 80 & Ennominae & Chiasmia emersaria & & (Walker, 1861) & S8 \\
\hline 81 & Ennominae & Chiasmia sp. & & & S3 \\
\hline 82 & Ennominae & Hyperythra lutea & & (Stoll, [1781]) & S3 \\
\hline 83 & Ennominae & Cleora alienaria & & (Walker, 1860) & S3 \\
\hline 84 & Geometrinae & Agathia laetata & & (Fabricius, 1794) & S1 \\
\hline 85 & Geometrinae & Agathia lycaenaria & & (Kollar, 1848) & S1 \\
\hline 86 & Geometrinae & Comibaena sp. & & & S3 \\
\hline 87 & Geometrinae & Dysphania militaris & & (Linnaeus, 1758) & S1 \\
\hline 88 & Geometrinae & Pingasa sp. & & & S7 \\
\hline 89 & Geometrinae & Thalassodes sp. & & & S3 \\
\hline 90 & Sterrhinae & Chrysocraspeda faganaria & & Guenée, [1858] & S3 \\
\hline 91 & Sterrhinae & Scopula emissaria & & (Walker, 1861) & $\mathrm{S3}$ \\
\hline 92 & Sterrhinae & Scopula sp. & & & S1 \\
\hline 93 & Sterrhinae & Traminda aventiaria & $\begin{array}{l}\text { Cross-Line } \\
\text { Wave Moth }\end{array}$ & (Guenée, [1858]) & $\mathrm{S} 2$ \\
\hline \multicolumn{6}{|c|}{ Superfamily Noctuoidea } \\
\hline \multicolumn{6}{|c|}{ Family Erebidae } \\
\hline 94 & Aganainae & Asota caricae & & (Fabricius, 1775) & S3 \\
\hline 95 & Arctiinae & Aloa lactinea & $\begin{array}{l}\text { Red Costate } \\
\text { Tiger Moth }\end{array}$ & (Cramer, [1777]) & S5 \\
\hline 96 & Arctiinae & Amata passalis & & (Fabricius, 1781) & S3 \\
\hline 97 & Arctiinae & Amerila astreus & & (Drury, 1773) & S3 \\
\hline
\end{tabular}


Pattanaik N et al. (2021). Not Sci Biol 13(3):10934

\begin{tabular}{|c|c|c|c|c|c|}
\hline 98 & Arctiinae & $\begin{array}{l}\text { Creatonotos gangis- } \\
\text { interrupta complex }\end{array}$ & & & S8 \\
\hline 99 & Arctiinae & Creatonotos transiens & & (Walker, 1855) & S3 \\
\hline 100 & Arctiinae & Kathasp. & & & S4 \\
\hline 101 & Arctiinae & Micraloa lineola & & (Fabricius, 1793) & S3 \\
\hline 102 & Arctiinae & Miltochrista sp. & & & S1 \\
\hline 103 & Arctiinae & Olepa ricini & & Fabricius, 1775 & S1 \\
\hline 104 & Arctiinae & Utetheisa sp. & & & S3 \\
\hline 105 & Boletobiinae & Eublemma accedens & & $\begin{array}{c}\text { (Felder \& } \\
\text { Rogenhofer, } \\
1874)\end{array}$ & S3 \\
\hline 106 & Boletobiinae & Zurobata vacillans & & (Walker, 1864) & $\mathrm{S} 2$ \\
\hline 107 & Calpinae & Calyptra sp. & & & $\mathrm{S} 2$ \\
\hline 108 & Calpinae & Eudocima hypermnestra & & (Cramer, 1780) & $\mathrm{S} 2$ \\
\hline 109 & Calpinae & Eudocima materna & $\begin{array}{c}\text { Dot Underwing } \\
\text { Moth }\end{array}$ & (Linnaeus, 1767) & S3 \\
\hline 110 & Eulepidotinae & Anticarsia irrorata & & (Fabricius, 1781) & S3 \\
\hline 111 & Erebinae & Achaea janata & & (Linnaeus, 1758) & S3 \\
\hline 112 & Erebinae & Bastilla simillima & & (Guenée, 1852) & S3 \\
\hline 113 & Erebinae & Chalciope mygdon & & (Cramer, [1777]) & S6 \\
\hline 114 & Erebinae & Dysgonia angularis & & $\begin{array}{c}\text { (Boisduval, } \\
1833 \text { ) } \\
\end{array}$ & $\mathrm{S} 2$ \\
\hline 115 & Erebinae & Dysgonia torrida & Jigsaw Moth & (Guenée, 1852) & S1 \\
\hline 116 & Erebinae & Ercheia sp. & & & S3 \\
\hline 117 & Erebinae & Erebus hieroglyphica & & (Drury, 1773) & S1 \\
\hline 118 & Erebinae & Grammodes geometrica & & (Fabricius, 1775) & S6 \\
\hline 119 & Erebinae & Hulodes sp. & & & S3 \\
\hline 120 & Erebinae & Mocis frugalis & $\begin{array}{c}\text { Sugarcane } \\
\text { Looper Moth }\end{array}$ & (Fabricius, 1775) & S3 \\
\hline 121 & Erebinae & Mocis undata & $\begin{array}{l}\text { Brown-Striped } \\
\text { Semi-Looper }\end{array}$ & (Fabricius, 1775) & S3 \\
\hline 122 & Erebinae & Pericyma cruegeri & $\begin{array}{l}\text { Poinciana } \\
\text { Looper Moth }\end{array}$ & (Butler, 1886) & S3 \\
\hline 123 & Erebinae & Serrodes partita & Catapult Moth & (Fabricius, 1775) & S3 \\
\hline 124 & Erebinae & Spirama sp. & & & S1 \\
\hline 125 & Erebinae & Thyas coronata & $\begin{array}{c}\text { Yellow } \\
\text { Underwing } \\
\text { Moth }\end{array}$ & (Fabricius, 1775) & S5 \\
\hline 126 & Lymantriinae & Arctornis cygna & & (Moore, 1879) & S3 \\
\hline 127 & Lymantriinae & Arctornis sp. & & & S8 \\
\hline 128 & Lymantriinae & Artaxa digramma & & $\begin{array}{c}\text { (Boisduval, } \\
1844)\end{array}$ & $\mathrm{S} 2$ \\
\hline 129 & Lymantriinae & Euproctis sp. & & & S3 \\
\hline
\end{tabular}


Pattanaik N et al. (2021). Not Sci Biol 13(3):10934

\begin{tabular}{|c|c|c|c|c|c|}
\hline 130 & Lymantriinae & Lymantria ampla & & Walker, 1855 & S1 \\
\hline 131 & Lymantriinae & Olene mendosa & $\begin{array}{l}\text { Brown Tussock } \\
\text { Moth }\end{array}$ & Hübner, 1823 & S3 \\
\hline 132 & Lymantriinae & Orvasca subnotata & & Walker, 1865 & S3 \\
\hline 133 & Lymantriinae & Perina nuda & $\begin{array}{l}\text { Banyan Tussock } \\
\text { Moth }\end{array}$ & (Fabricius, 1787) & S3 \\
\hline 134 & Pangraptinae & Egnasia ephyrodalis & & Walker, 1858 & S2 \\
\hline 135 & Scoliopteryginae & Anomis flava & $\begin{array}{l}\text { Cotton Looper } \\
\text { Moth }\end{array}$ & (Fabricius, 1775) & S1 \\
\hline \multicolumn{6}{|c|}{ Family Nolidae } \\
\hline 136 & Chloephorinae & Carea angulata & & (Fabricius, 1793) & S1 \\
\hline 137 & Eariadinae & Earias luteolaria & & Hampson, 1891 & S8 \\
\hline 138 & Eariadinae & Earias vittella & $\begin{array}{c}\text { Spotted } \\
\text { Bollworm Moth }\end{array}$ & Fabricius, 1794 & S3 \\
\hline 139 & Eligminae & Selepa celtis & & Moore, [1858] & S2 \\
\hline 140 & Nolinae & Nola sp. & & & S3 \\
\hline 141 & Risobinae & Risoba repugnans & & (Walker, 1865) & S8 \\
\hline \multicolumn{6}{|c|}{ Family Noctuidae } \\
\hline 142 & Acontiinae & Acontia lucida & $\begin{array}{l}\text { Pale Shoulder } \\
\text { Moth }\end{array}$ & (Hufnagel, 1766) & S1 \\
\hline 143 & Acontiinae & Acontia marmoralis & & (Fabricius, 1794) & S1 \\
\hline 144 & Acontiinae & Naranga aenescens & & Moore, 1881 & S3 \\
\hline 145 & Agaristinae & Episteme sp. & & & S1 \\
\hline 146 & Catocalinae & Gesonia obeditalis & & Walker, [1859] & S6 \\
\hline 147 & Condicinae & Condica illecta & & (Walker, 1865) & S3 \\
\hline 148 & Eriopinae & Callopistria sp. & & & S3 \\
\hline 149 & Hadeninae & Mythimna separata & & (Walker, 1865) & S1 \\
\hline 150 & Hadeninae & Mythimnasp. & & & S2 \\
\hline 151 & Heliothinae & Helicoverpa armigera & & $\begin{array}{l}\text { (Hübner, } \\
[1808])\end{array}$ & S3 \\
\hline 152 & Noctuinae & Polytela gloriosae & Lily Moth & (Fabricius, 1781) & S9 \\
\hline 153 & Noctuinae & Spodoptera litura & $\begin{array}{l}\text { Tobacco } \\
\text { Cutworm Moth }\end{array}$ & (Fabricius, 1775) & $S 10$ \\
\hline 154 & Plusiinae & Chrysodeixis eriosoma & & $\begin{array}{l}\text { (Doubleday, } \\
1843)\end{array}$ & S3 \\
\hline \multicolumn{6}{|c|}{$\begin{array}{l}\text { S1 to S10 Study Sites: [S1- Acharya Vihar, S2- Jaydev Vihar, S3- BJB Nagar, S4- Saheed Nagar, S5- Khandagiri, } \\
\text { S6- Pokhariput, S7- Ghangapatna, S8- Dhauli, S9- Dalua, S10- Raghunathpur] }\end{array}$} \\
\hline
\end{tabular}



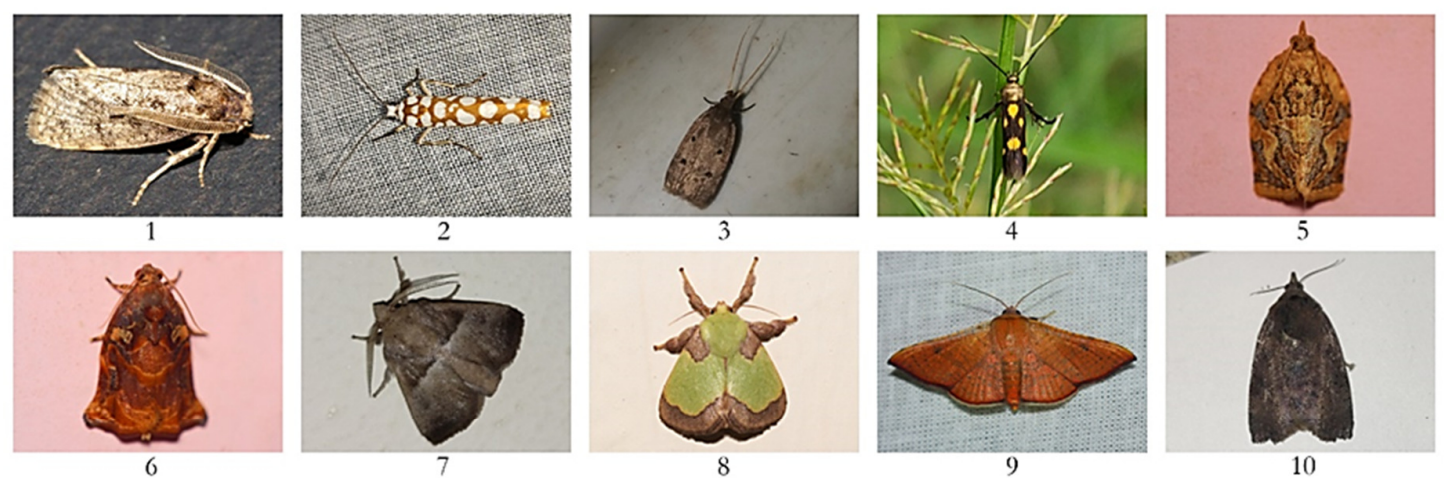

8

10

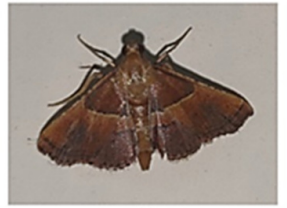

11

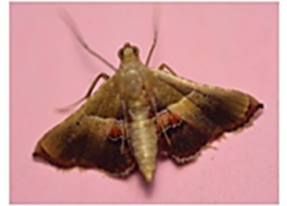

12

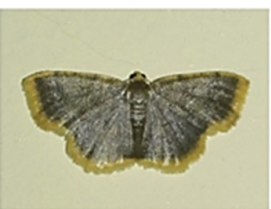

13

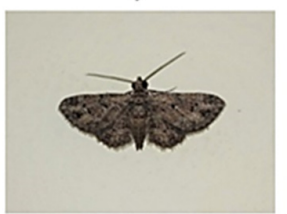

14

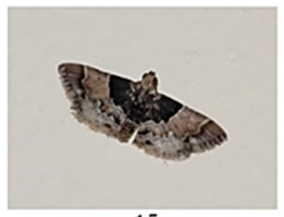

15

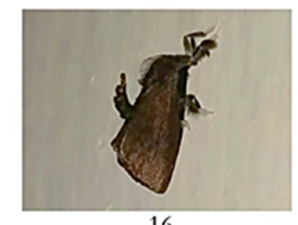

16

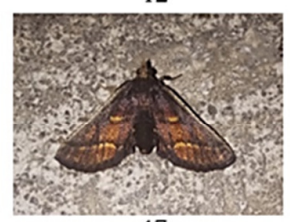

17

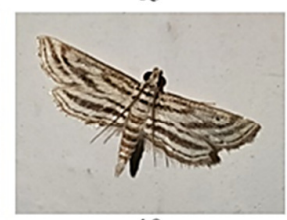

18

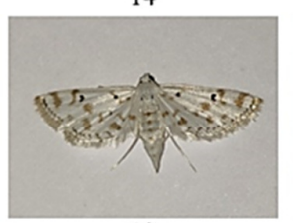

19
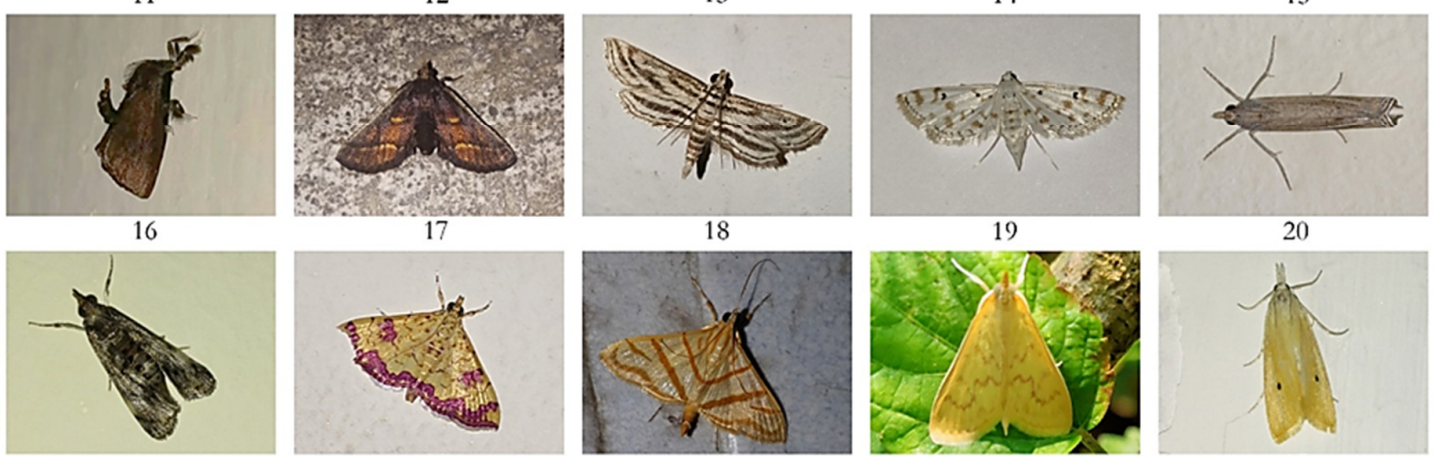

21

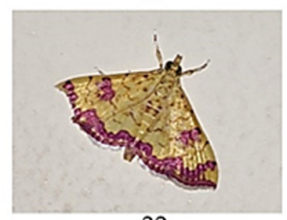

22

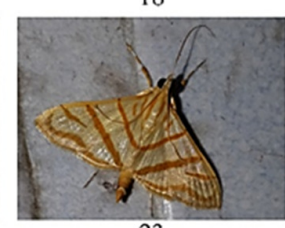

23

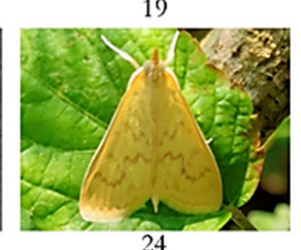

24
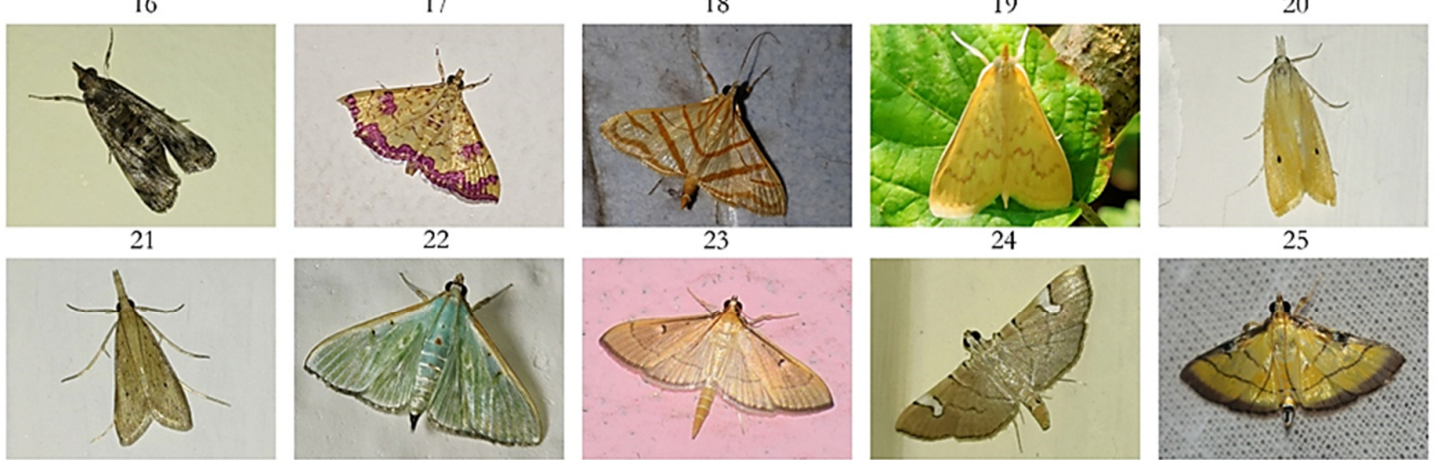

26

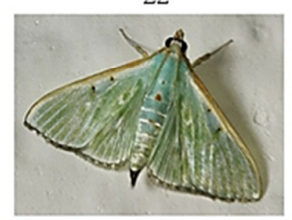

27

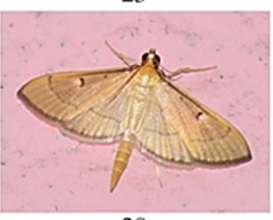

28

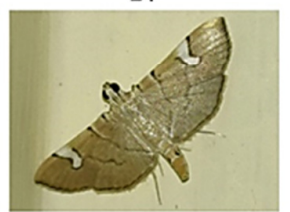

29

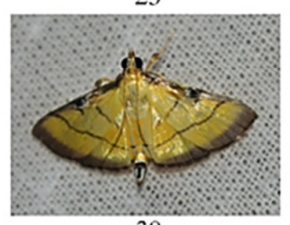

30

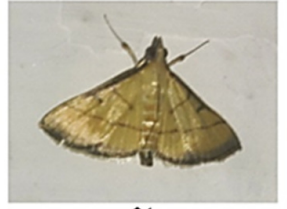

31

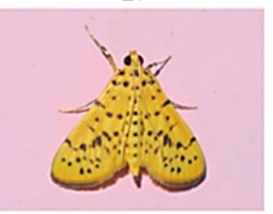

32

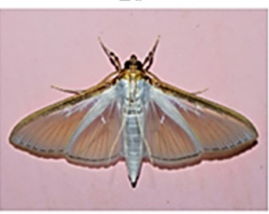

$3 \hat{3}$

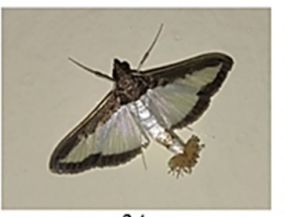

34

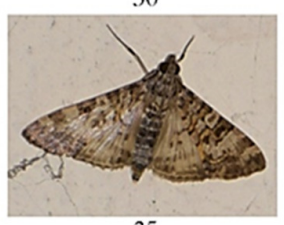

35

Plate 1. 1- Acrolophus sp.; 2- Atteva sp.; 3- Lecithocera sp.; 4- Eretmocera impactella; 5- Adoxophyes fasciculana; 6- Archips micaceana; 7- Aphendala tripartita; 8- Parasa sp.; 9- Banisia sp.; 10- Hyblaea sp.; 11- Endotricha mesenterialis, 12- Endotricha repandalis; 13- Hypsopygia sp.; 14- Pyralis manihotalis; 15Pyralis pictalis; 16- Sacada sp.; 17- Zitha torridalis; 18- Parapoynx fluctuosalis; 19- Parapoynx stagnalis; 20- Ancylolomia sp.; 21- Noorda blitealis; 22- Isocentris filalis; 23- Pagyda salvalis; 24- Paliga sp.; 25Scirpophaga incertulas; 26- Scirpophaga nivella; 27- Arthroschista hilaralis; 28- Bradina admixtalis, 29Chalcidoptera appensalis; 30- Cnaphalocrocis medinalis; 31 - Cnaphalocrocis ruralis; 32- Conogethes sp.; 33- Cydalima laticostalis; 34- Diaphania indica; 35- Dysallacta negatalis 


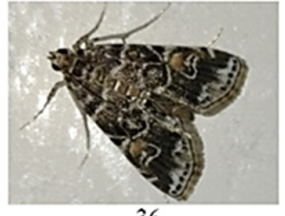

36

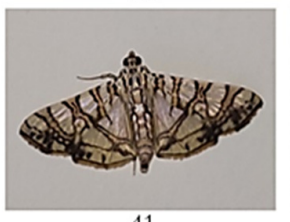

41

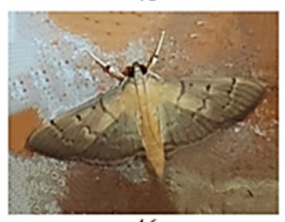

46

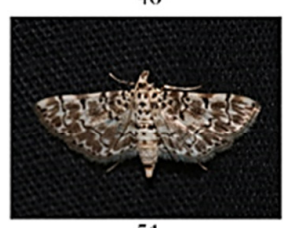

51

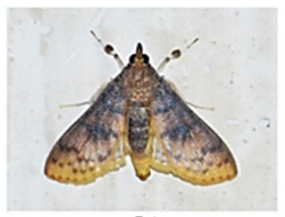

56

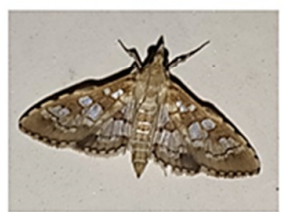

61

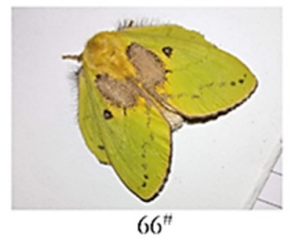

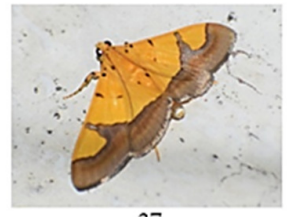

37

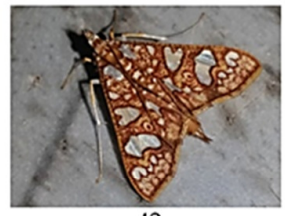

42

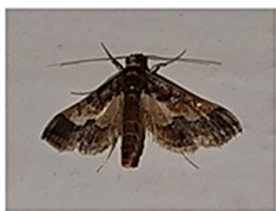

47

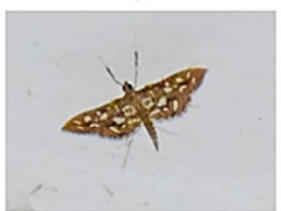

52

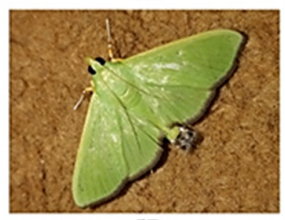

57

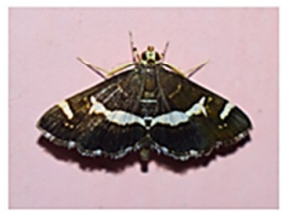

62

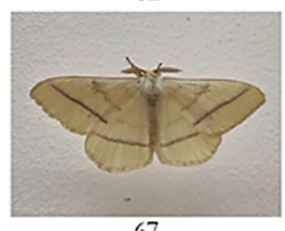

67

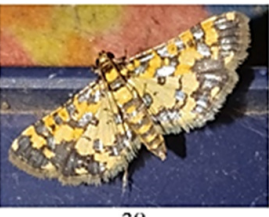

38

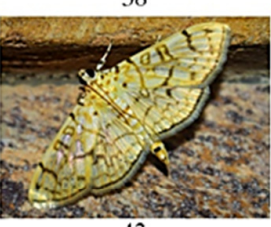

43

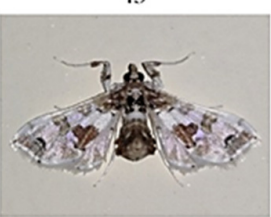

48

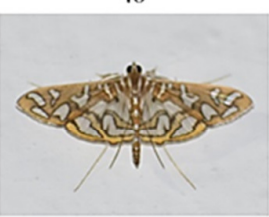

53

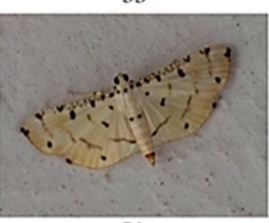

58

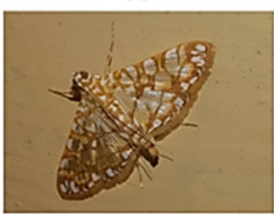

63

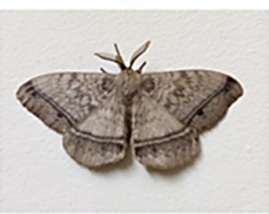

68

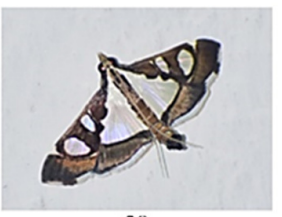

39

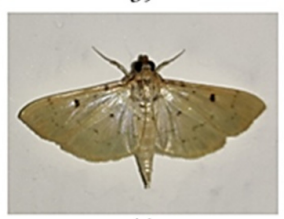

44

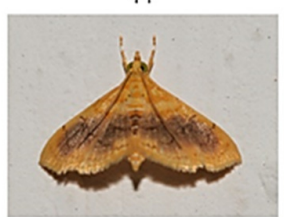

49

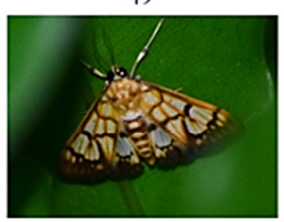

54

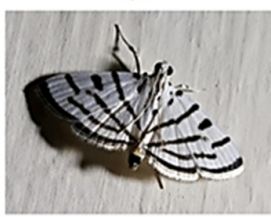

59

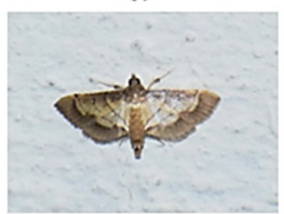

64

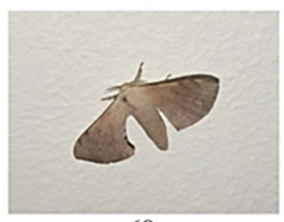

69

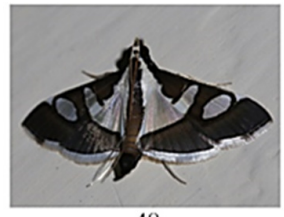

40

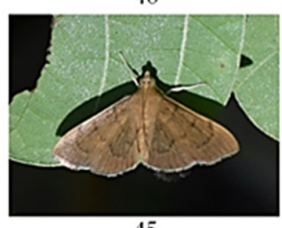

45

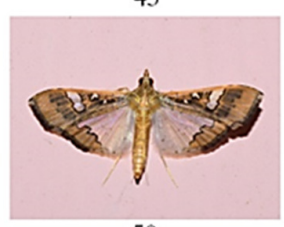

50

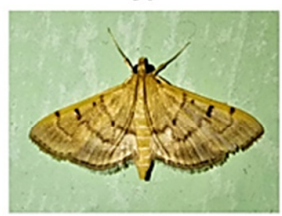

$55^{*}$

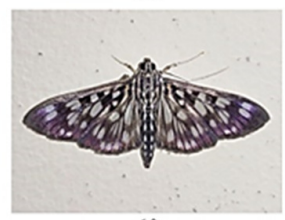

60

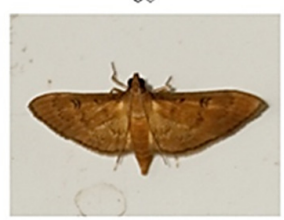

65

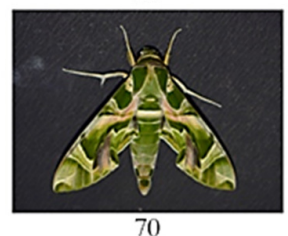

70

Plate 2.36- Elophila difflualis; 37- Endocrossis flavibasalis; 38- Eurrhyparodes tricoloralis; 39- Glyphodes actorionalis; 40- Glyphodes bicolor; 41- Glyphodes caesalis; 42- Glyphodes canthusalis; 43- Haritalodes derogata; 44- Herpetogramma basalis; 45- Herpetogramma phaeopteralis; 46- Herpetogramma rudis; 47Hydriris ornatalis; 48- Leucinodes orbonalis; 49- Mabra eryxalis; 50- Maruca vitrata; 51- Metoeca foedalis; 52- Nausinoe geometralis; 53- Nausinoe perspectata; 54- Nosophora sp.; 55- Omiodes indicata; 56Pachynoa sp.; 57- Parotis sp.; 58- Pycnarmon cribrata; 59- Pycnarmon virgatalis; 60- Pygospila tyres; 61Sameodes cancellalis; 62- Spoladea recurvalis; 63- Synclera traducalis; 64- Syngamia latimarginalis; 65Tatobotys biannulalis; 66- Trabala vishnou; 67- Eupterote bifasciata; 68- Eupterote undata; 69- Trilocha varians; 70 - Daphnis nerii

*Picture Credits: B. Swarup Kumar Subudhi; " Picture Credits: Ananya Kashyap 


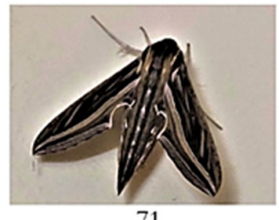

71

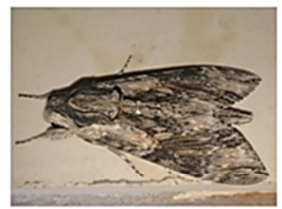

76

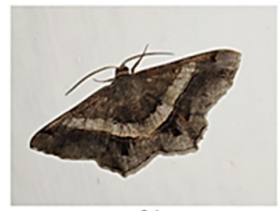

81

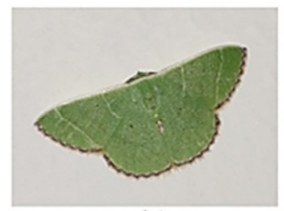

86

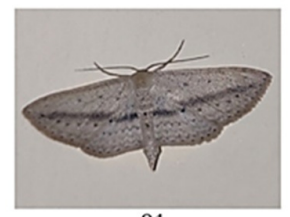

91

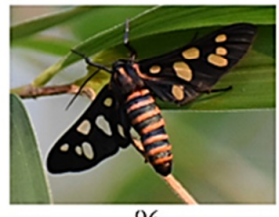

96

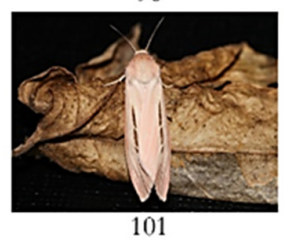

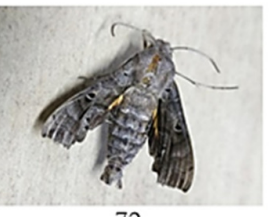

72

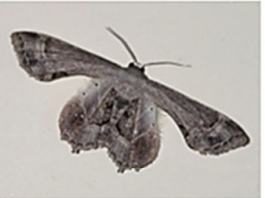

77

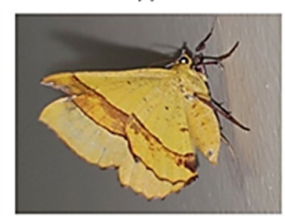

82

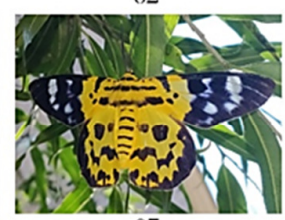

87

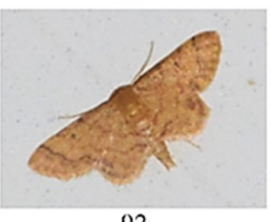

92

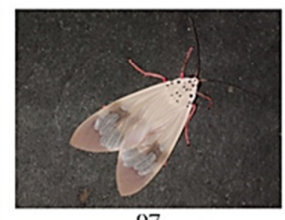

97

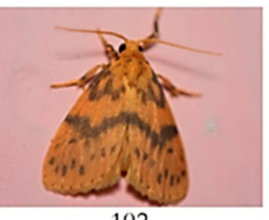

102

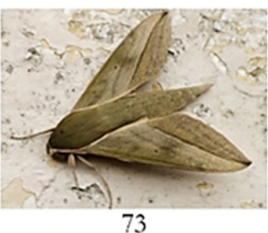

73

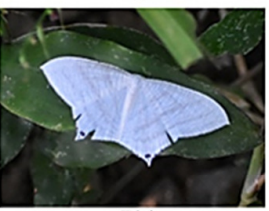

$78^{\wedge}$

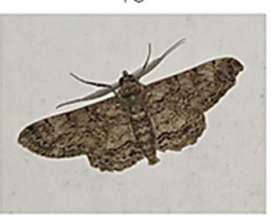

83

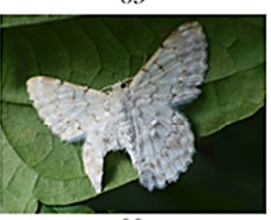

88

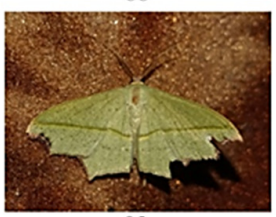

93

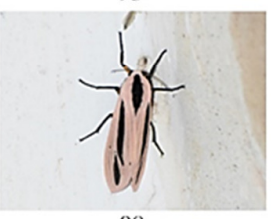

98

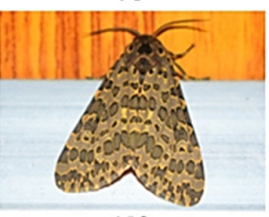

103
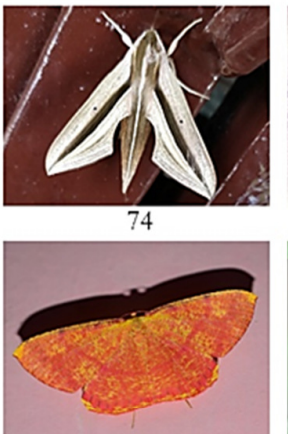

79

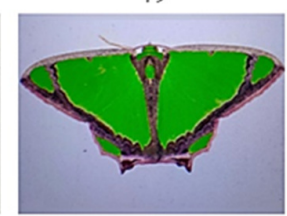

84

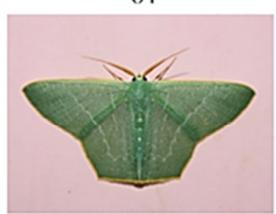

89

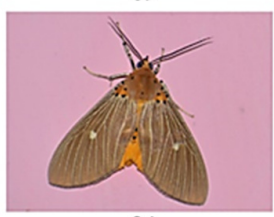

94

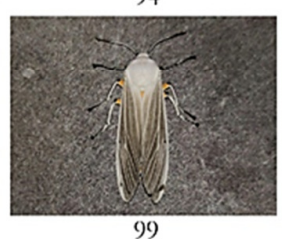

99

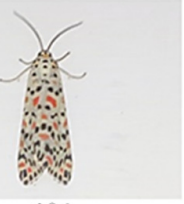

104

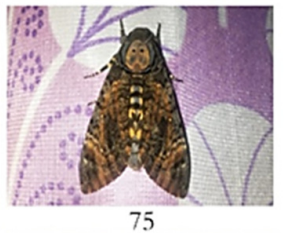

75

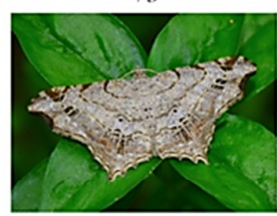

80

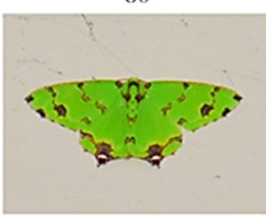

85

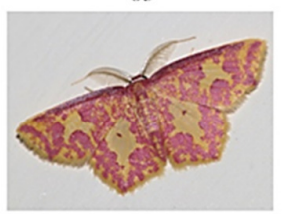

90
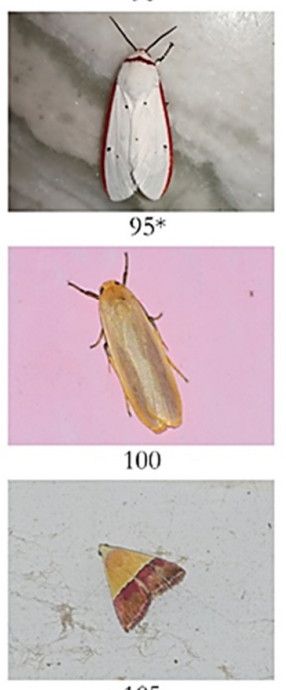

105

Plate 3.71- Hippotion celerio; 72- Macroglossum sp.; 73- Theretra lucasii; 74- Theretra oldenlandiae; 75Acherontia styx;76- Psilogramma sp.;77- Phazaca theclata;78- Micronia aculeata;79- Eumelea ludovicata; 80- Chiasmia emersaria; 81- Chiasmia sp.; 82- Hyperythra lutea; 83- Cleora alienaria; 84- Agathia laetata; 85- Agathia lycaenaria; 86- Comibaena sp.; 87- Dysphania militaris; 88- Pingasa sp.; 89- Thalassodes sp.; 90- Chrysocraspeda faganaria; 91- Scopula emissaria; 92- Scopula sp.; 93- Traminda aventiaria; 94- Asota caricae; 95- Aloa lactinea; 96- Amata passalis; 97- Amerila astreus; 98- Creatonotos gangis-interrupta complex; 99- Creatonotos transiens; 100- Katha sp.; 101- Micraloa lineola; 102- Miltochrista sp.; 103Olepa ricini; 104- Utetheisa sp.; 105- Eublemma accedens

${ }^{\wedge}$ Picture Credits: Sabindra Kumar Samal; *Picture Credits: B. Swarup Kumar Subudhi 


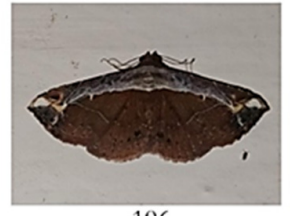

106

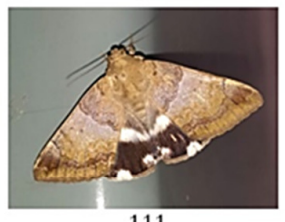

111

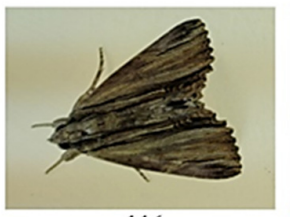

116

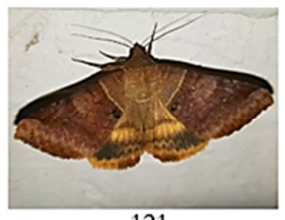

121

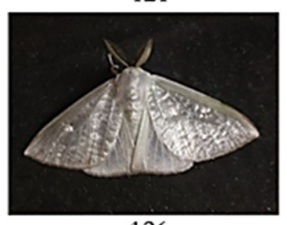

126

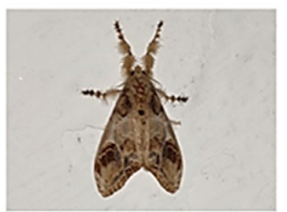

131

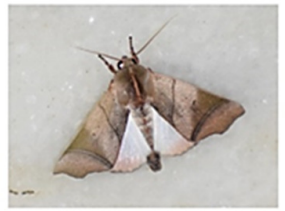

136

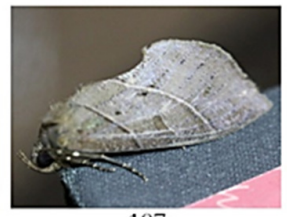

107

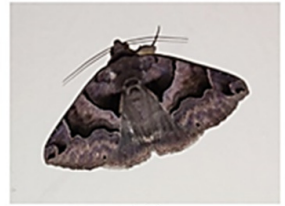

112

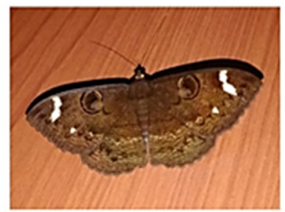

117

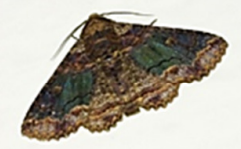

122

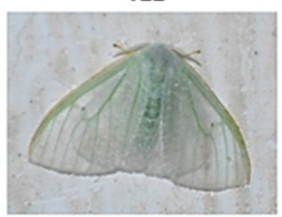

127

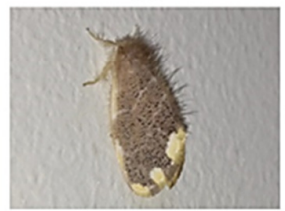

132

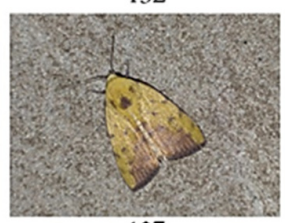

137

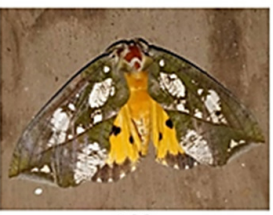

108

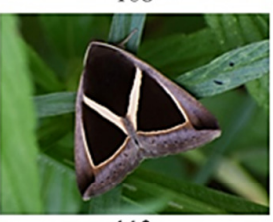

113

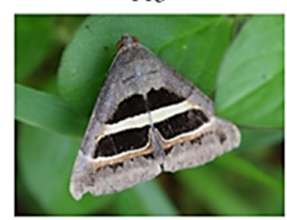

118

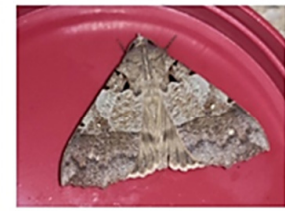

23

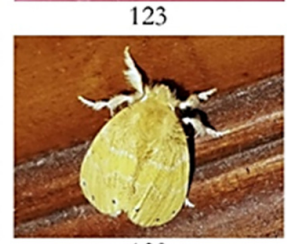

128

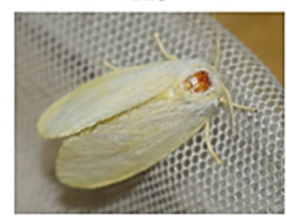

133

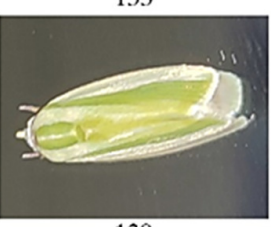

138

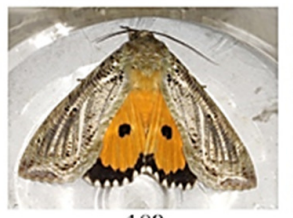

109

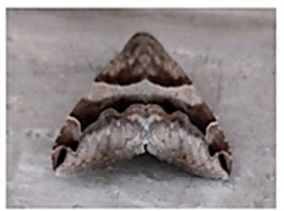

114

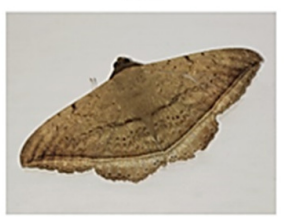

119

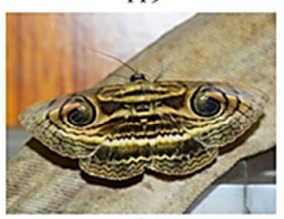

124

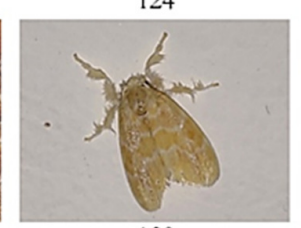

129

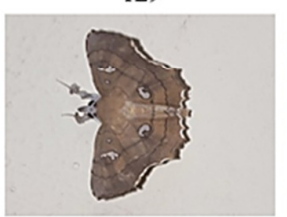

134

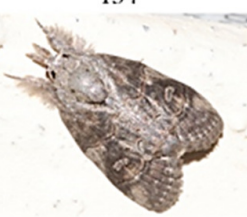

139

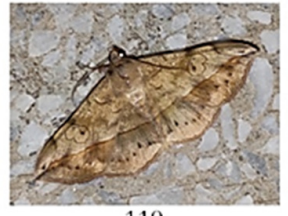

110

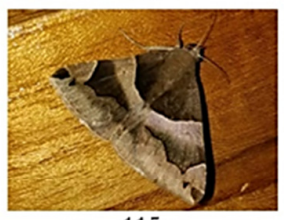

115

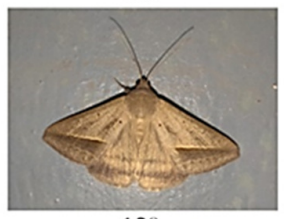

120
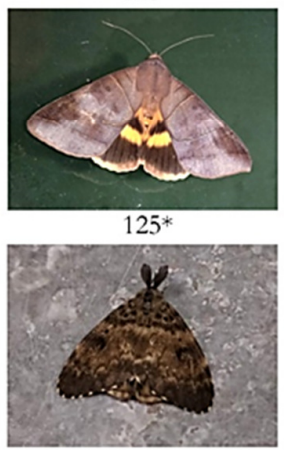

130

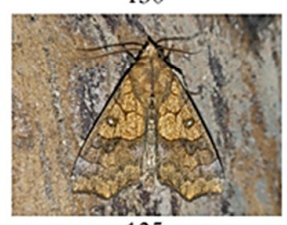

135

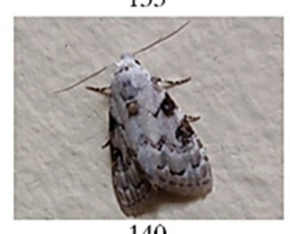

140

Plate 4. 106- Zurobata vacillans; 107- Calyptra sp.; 108- Eudocima hypermnestra; 109- Eudocima materna; 110- Anticarsia irrorata; 111- Achaea janata; 112- Bastilla simillima; 113- Chalciope mygdon; 114- Dysgonia angularis; 115- Dysgonia torrida; 116- Ercheia sp.; 117- Erebus hieroglyphica; 118Grammodes geometrica; 119- Hulodes sp.; 120- Mocis frugalis; 121- Mocis undata; 122- Pericyma cruegeri; 123- Serrodes partita; 124- Spirama sp.; 125- Thyas coronata; 126- Arctornis cygna; 127Arctornis sp.; 128- Artaxa digramma; 129- Euproctis sp.; 130- Lymantria ampla; 131- Olene mendosa; 132- Orvasca subnotata; 133- Perina nuda; 134- Egnasia ephyrodalis; 135- Anomis flava; 136- Carea angulata; 137- Earias luteolaria; 138- Earias vittella; 139- Selepa celtis; 140- Nola sp.

${ }^{*}$ Picture Credits: B. Swarup Kumar Subudhi 


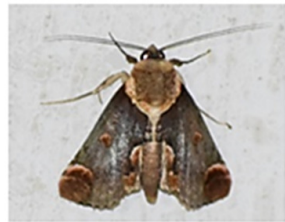

141

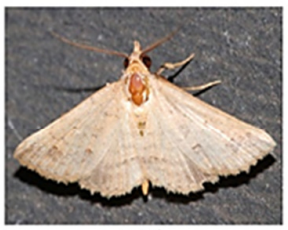

146

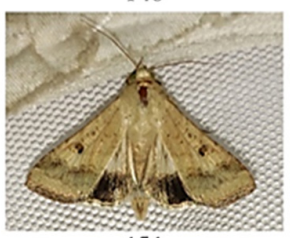

151

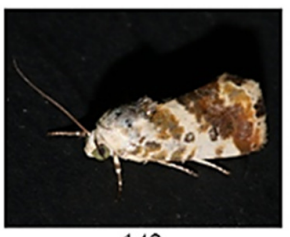

142

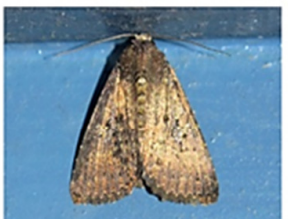

147

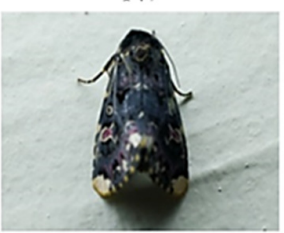

152

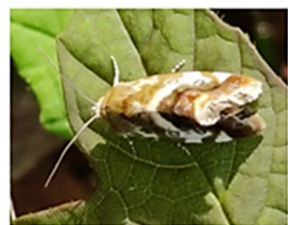

143

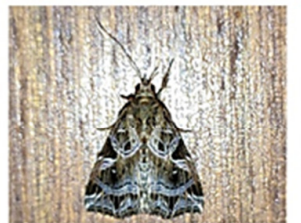

148

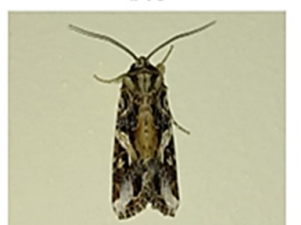

153

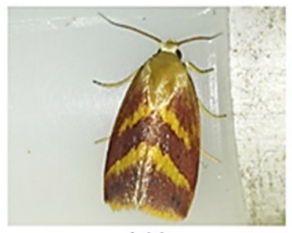

144

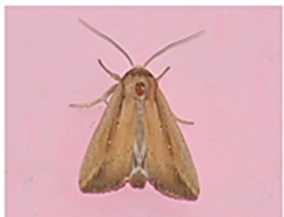

149

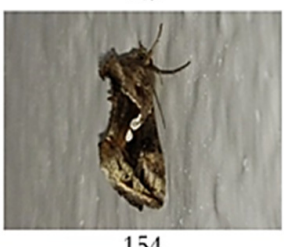

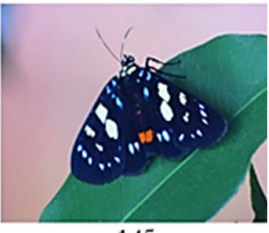

145

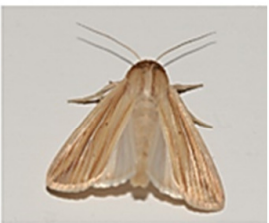

150

Plate 5. 141- Risoba repugnans; 142- Acontia lucida; 143- Acontia marmoralis; 144- Naranga aenescens; 145- Episteme sp.; 146- Gesonia obeditalis; 147- Condica illecta; 148- Callopistria sp.; 149- Mythimna separata; 150- Mythimna sp.; 151- Helicoverpa armigera; 152- Polytela gloriosae; 153- Spodoptera litura; 154- Chrysodeixis eriosoma

Here in this study, we have recorded 19 moth families being reported from the state of Odisha which includes 154 species under 129 genera. Out of these, 34 moths have been identified only up to the genus level, while the rest have been identified up to species level as indicated in Table 2. In the study, family Crambidae dominated in species diversity, composing $31.2 \%$ of the total species ( 48 species, 38 genera), followed by Erebidae composing 27.3\% ( 42 species, 37 genera), Geometridae making up for 9.7\% (15 species, 12 genera) and Noctuidae at $8.4 \%$ (13 species, 11 genera). The other families found in less numbers were in the following order of species diversity namely, Sphingidae with seven species in six genera (4.5\%), Pyralidae with seven species in five genera (4.5\%) and Nolidae with six species in five genera (3.9\%). Further, the families Limacodidae, Tortricidae and Uraniidae were represented by two species in two genera each while, Eupterotidae had one genus with two species and the rest eight families (Tineidae, Scythrididae Lasiocampidae, Attevidae, Thyrididae, Bombycidae, Hyblaeidae and Lecithoceridae) were found with a single species in each (Figure 4). 


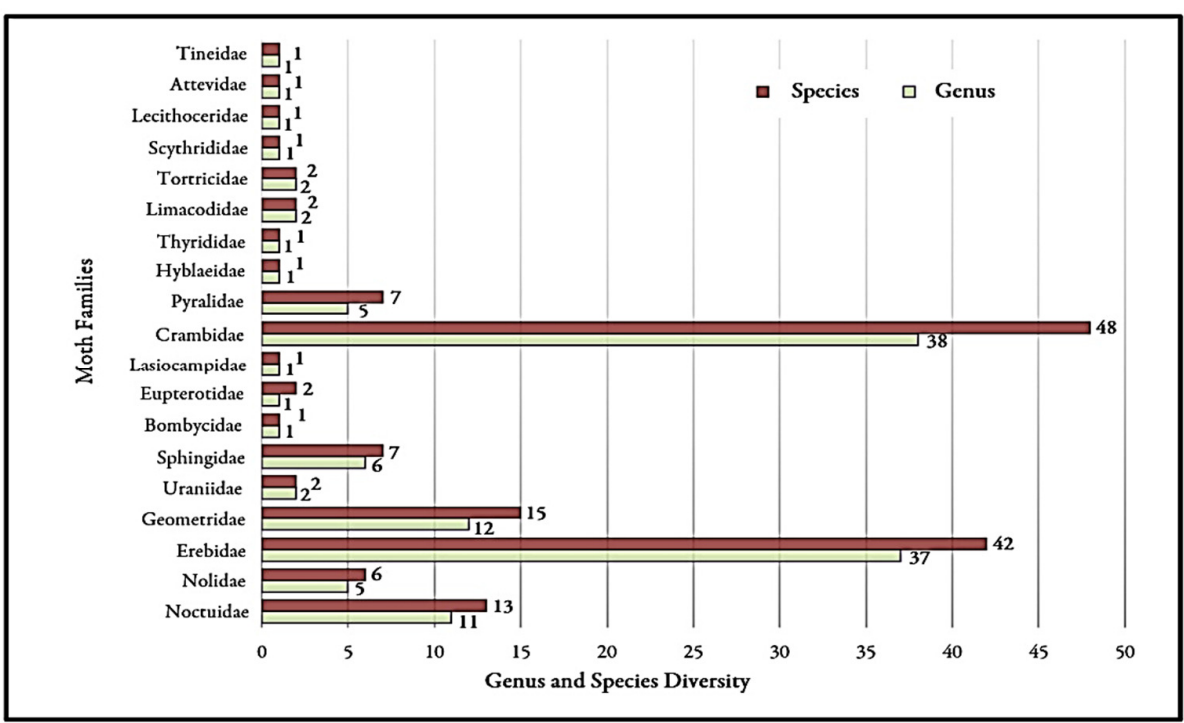

Figure 4. Graph denoting genus and species diversity in observed moth families

The study reveals a specific pattern of presence of moth families across various months in a year. Moths from the family Crambidae were found all throughout the year, followed by Erebidae and Geometridae which were recorded in around ten months across the year. Noctuidae, Pyralidae and Sphingidae were observed in seven different months of the year. Nolidae and Bombycidae moths were seen in around four to six months in different seasons. The families of moths which were less found were reported in one or two months in the whole year. These were Uraniidae, Eupterotidae, Lasiocampidae, Scythrididae, Lecithoceridae, Thyrididae, Tineidae, Hyblaeidae, Attevidae, Limacodidae and Tortricidae (Table 3). While most moths that have been found were crepuscular in their time of activity and presence, day-flying moths like Episteme sp. and Dysphania militaris were also recorded amongst macrolepidoptera.

Table 3. Presence of different moth families across different months

\begin{tabular}{|c|c|c|c|c|c|c|c|c|c|c|c|c|c|}
\hline Superfamily & Family & Jan & Feb & Mar & Apr & May & Jun & Jul & Aug & Sep & Oct & Nov & Dec \\
\hline Tineoidea & Tineidae & & & & & + & & & & & & & \\
\hline Yponomeutoidea & Attevidae & & & & & & & + & & & & & \\
\hline \multirow{2}{*}{ Gelechioidea } & Lecithoceridae & & & & & & & & & & + & & \\
\hline & Scythrididae & & & & & & + & + & & & & & \\
\hline Tortricoidea & Tortricidae & & & + & & & & & + & + & & & \\
\hline Zygaenoidea & Limacodidae & & & & & & & & + & & + & + & \\
\hline Thyridoidea & Thyrididae & & & & & & & & & & + & + & \\
\hline Hyblaeoidea & Hyblaeidae & & & & & & & & + & & & & \\
\hline \multirow{2}{*}{ Pyraloidea } & Pyralidae & + & & + & & & + & + & + & + & + & & \\
\hline & Crambidae & + & + & + & + & + & + & + & + & + & + & + & + \\
\hline Lasiocampoidea & Lasiocampidae & & & & & + & & & & & & + & \\
\hline \multirow{3}{*}{ Bombycoidea } & Eupterotidae & & & & & + & & + & & & + & & \\
\hline & Bombycidae & + & & & & & + & + & + & & & & \\
\hline & Sphingidae & + & & & & + & + & + & + & + & & + & \\
\hline \multirow{2}{*}{ Geometroidea } & Uraniidae & & & & & & & & + & & & + & \\
\hline & Geometridae & + & + & + & + & + & + & & + & + & + & + & \\
\hline \multirow{3}{*}{ Noctuoidea } & Erebidae & + & + & & & + & + & + & + & + & + & + & + \\
\hline & Nolidae & + & & & & & + & + & + & & + & + & \\
\hline & Noctuidae & + & + & & + & & + & + & & + & + & & \\
\hline
\end{tabular}


The month of August recorded the highest diversity of moths from 11 different families out of all 19 families reported in the study. July and October recorded a considerably higher number of moths with ten families reported in each month. Moths from families Crambidae, Geometridae and Erebidae were found across most seasons while others like Limacodidae, Thyrididae and Lecithoceridae were only seen during autumn and winters. The families represented by a greater number of moth species were mostly found around monsoon (Figure 5). Hence from the study, it can be said that the diversity of moths is quite rich in Odisha. Since the present inventory relied mostly on opportunistic findings and seasonal surveys of 18 months yet reports a diversity of 19 families with 154 species from a single district of Khordha, it is contemplated that further studies in detail with intensive light trapping sessions can reveal the actual diversity of the eastern state of Odisha.

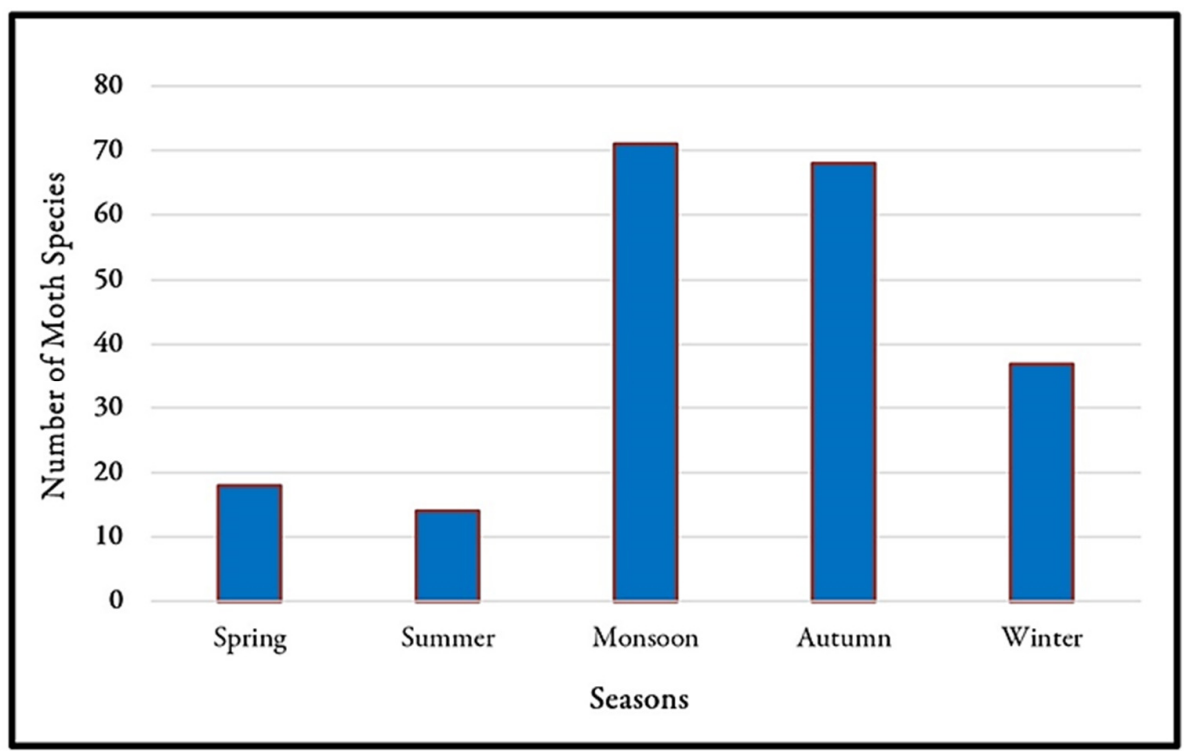

Figure 5. Seasonal distribution of recorded moth species

\section{Conclusions}

The study compiles a preliminary moth diversity of the city of Bhubaneswar and adjoining outskirts, recording a total of 154 moths in 19 families. It can be said that the presence of various moth species in any particular landscape is related to the different types of vegetation of a region, cropping seasons, the flowering of plants and various other factors controlling their diversity and abundance. Hence, this suggests that the moth diversity of the state is quite rich as evident from a preliminary survey in a single district and needs to be extensively studied, to gather more information about their present status for further conservation. Many species found in the study could be keyed only till the genus level while many other unidentified moths await proper taxonomic studies and documentation. As the state of Odisha is rich in forest cover and has diverse biogeographic zones from the East Coast to Deccan Peninsula including tropical dry deciduous and semievergreen forest types, therefore it can be easily speculated that the moth fauna of the state is unique and rich as found from the present sample study of one district.

It is evident that with further intensive studies in the other parts of the state, the moth diversity can be explored in greater detail in relation to the biogeographic regions and vegetation types across the state. The results of the present survey indicate a diverse population of moths present in the landscape of Odisha with 19 families reported, characterized majorly by Crambidae, Erebidae, Geometridae and Noctuidae. The presence 
of families less encountered like, Tineidae, Attevidae, Lecithoceridae and Hyblaeidae also indicate that moths can be easily considered as bioindicators for particular regions when correlated to their presence in particular forest types or habitat. We also suggest that since inventorying is necessary for conservation of a taxon, more biodiversity assessments need to be done on these largely nocturnal lepidopterans. Along with natural history documentation, scientific records of the same can also reveal more information about interactions with plants and their vital role which they play in the ecosystem as indicators, pollinators and pests, other than the usual importance given to few silk moths for economic benefits. It would be further interesting to compare the diversity from urban areas like the present study locations with forested areas which stand unaffected by the city light pollution, which affects moths and their natural navigation in a huge way.

\section{Authors' Contributions}

The study was supervised by DP. AS has majorly contributed towards photography of the moths while NP identified the species and drew maps and figures. AS and NP put together the data into tables and charts. All authors contributed together for fieldwork and preparation of the manuscript, while review and editing were done by DP.

All authors read and approved the final manuscript.

Ethical approval (for researches involving animals or humans)

For the present study, none of the moths were collected or killed and therefore live photography of the moths was done as presented in the image plates.

\section{Acknowledgements}

The authors would like to thank the Scientist-in Charge, Regional Museum of Natural History, Bhubaneswar for necessary permissions to carry out baseline observations. Thanks are due to HOD, Zoology Department, B.J.B. (Autonomous) College, Bhubaneswar for kind permissions to conduct studies along with the regular curriculum. We also thank Mr. B. Swarup Kumar Subudhi for his generous help in contributing moth photographs from various places across the city and accompanying in several field trips with much enthusiasm. Acknowledgments are also due to the staff of RMNH, Bhubaneswar who helped us to set up the light traps and arrange for other facilities during the study. We would extend our heartfelt thanks to various scientists, researchers working in this discipline and enthusiasts on various social platforms, who have helped us in identification and friends \& family who encouraged us to put up the work together. This research received no specific grant from any funding agency in the public, commercial, or not-for-profit sectors.

\section{Conflict of Interests}

The authors declare that there are no conflicts of interest related to this article.

\section{References}

Alfred JRB, Das AK, Sanyal AK (1998). Faunal Diversity in India. ENVIS Centre Zoological Survey of India, Kolkata pp 311-318. 
Arora GS (2000). Studies on some Indian Pyralid species of Economic Importance. Part I. Crambinae, Schoenobiinae, Nymphulinae, Phycitinae and Galleriinae (Lepidoptera: Pyralidae). Records of Zoological Survey of India, Zoological Survey of India, Calcutta. Occasional Paper No. 181, i-vii, 1-169.

Bell TRD, Scott FB (1937). Fauna of British India, including Ceylon and Burma. Moths-Volume 5, Sphingidae. Taylor and Francis, London.

Chandra K, Nema DK (2007). Fauna of Madhya Pradesh (including Chhattisgarh) Part-I, State Fauna Series, Zoological Survey of India, Kolkata.

Devoto M, Bailey S, Memmott J (2011). The night shift: nocturnal pollen-transport networks in a boreal pine forest. Ecological Entomology 36:25-35. https://doi.org/10.1111/j.1365-2311.2010.01247.x

Dey P, Joshi K, Uniyal VP (2018). Common Moths of WII. Wildlife Institute of India, Dehradun.

Dey P, Uniyal VP, Sanyal AK (2015). Moth Assemblages (Lepidoptera: Heterocera) as a potential conservation tool for biodiversity monitoring - Study in Western Himalayan protected areas. Indian Forester 141(9):985-992.

Envis Centre of Odisha (2020). ENVIS Centre of Odisha's State of Environment. Retrieved 2021 February 2 from http://www.orienvis.nic.in/

Faunal Diversity of India (2020). ENVIS Centre on Faunal Diversity. Retrieved 2021 February 2 from http://www.zsienvis.nic.in/

Gurule S, Nikam S (2013). The moths (Lepidoptera: Heterocera) of northern Maharashtra: a preliminary checklist. Journal of Threatened Taxa 5(12):4693-4713. http://dx.doi.org/10.11609/JoTT.o2555.4693-713

Hampson GF (1892). The Fauna of British India including Ceylon and Burma, Moths. Taylor and Francis (Volume 1), London.

Hampson GF (1894). The Fauna of British India including Ceylon and Burma, Moths. Taylor and Francis (Volume 2), London.

Hampson GF (1895). The Fauna of British India including Ceylon and Burma, Moths. Taylor and Francis (Volume 3), London.

Hampson GF (1896). The Fauna of British India including Ceylon and Burma, Moths. Taylor and Francis (Volume 4), London.

Holloway JD (1985). The Moths of Borneo (Part 14) - Noctuidae: Euteliinae, Stictopterinae, Plusiinae, Pantheinae. Southdene Sdn Bhd, Kuala Lumpur.

Holloway JD (1986). The Moths of Borneo (Part 1) - Key to Families: Cossidae, Metarbelidae, Ratardidae, Dudgeoneidae, Epipyropidae and Limacodidae. Southdene Sdn Bhd, Kuala Lumpur.

Holloway JD (1987). The Moths of Borneo (Part 3) - Lasiocampidae, Eupterotidae, Bombycidae, Brahmaeidae, Saturniidae, Sphingidae. Malaysian Nature Society, Kuala Lumpur.

Holloway JD (1988). The Moths of Borneo (Part 6) - Arctiidae: Syntominae, Euchomiinae, Arctiinae, Aganainae (to Noctuidae). Malaysian Nature Society, Kuala Lumpur.

Holloway JD (1989). The Moths of Borneo (Part 12) - Noctuidae: Noctuinae, Heliothinae, Hadeninae, Acronictinae, Amphipyrinae, Agaristinae. Southdene Sdn Bhd, Kuala Lumpur.

Holloway JD (1993). The Moths of Borneo (Part 11) - Geometridae: Ennominae. Southdene Sdn Bhd, Kuala Lumpur.

Holloway JD (1996). The Moths of Borneo (Part 9) - Geometridae (Incl. Orthostixini): Oenochrominae, Desmobathrinae, Geometrinae, Ennominae addenda. Southdene Sdn Bhd, Kuala Lumpur.

Holloway JD (1997). The Moths of Borneo (Part 10) - Geometridae: Sterrhinae, Larentiinae, Addenda to other subfamilies. Southdene Sdn Bhd, Kuala Lumpur.

Holloway JD (1998). The Moths of Borneo (Part 8) - Castniidae, Callidulidae, Drepanidae, Uraniidae. Southdene Sdn Bhd, Kuala Lumpur.

Holloway JD (1999). The Moths of Borneo (Part 5) - Lymantriidae. Southdene Sdn Bhd, Kuala Lumpur.

Holloway JD (2003). The Moths of Borneo (Part 18) - Nolidae. Malaysian Nature Society, Kuala Lumpur.

Holloway JD (2011). The Moths of Borneo (Part 2) - Phaudidae, Himantopteridae and Zygaenidae; revised and annotated checklist. Southdene Sdn Bhd, Kuala Lumpur.

HOSTS (2020). HOSTS - a Database of the World's Lepidopteran Hostplants. Natural History Museum. Retrieved 2021 February 2 from https://www.nhm.ac.uk/

iNaturalist (2020). iNaturalist. Retrieved 2021 February 2 from https://www.inaturalist.org/

Insect Pests (2020). Insects in Indian Agroecosystems. National Bureau of Agricultural Insect Resources. Retrieved 2021 February 2 from https://databases.nbair.res.in/ 
Jena SK, Singh AP, De K (2018). Diversity of moths (Insecta: Lepidoptera) in the Gupteswar proposed reserve forest of the Eastern Ghat Hill, Koraput, Odisha, India: A preliminary study. Egyptian Academic Journal of Biological Sciences 11(3):11-17. https://dx.doi.org/10.21608/eajb.2018.11677

Kar D, Kuanar A, Ray A, Gaur M, Pattanaik B, Mishra B (2020). Genetic diversity of Brinjal fruit and shoot borer (BSFB) population of Odisha, India. Iranian Journal of Science and Technology, Transactions A: Science 45:135-144. https://doi.org/10.1007/s40995-020-00997-y

Khordha Web Portal (2021). Map of district. Retrieved 2021 February 2 from https://khordha.nic.in/

Kononenko SV, Pinratana A (2013). Moths of Thailand Vol. 3, Part 2. Noctuoidea. An Illustrated Catalogue of Erebidae, Nolidae, Euteliidae, and Noctuidae (Insecta: Lepidoptera) in Thailand. Brothers of Saint Gabriel, Thailand.

Kristensen NP (1999). Lepidoptera, Moths and Butterflies. Vol. 1: Evolution, Systematics, and Biogeography. In: M. Fischer, Handbook of Zoology 4. Arthropoda: Insecta, part 35. Walter de Gruyter, Berlin \& New York pp 491.

Kristensen NP, Scoble MJ, Karsholt O (2007). Lepidoptera phylogeny and systematics: the state of inventorying moth and butterfly diversity. In: Zhang Z-Q, Shear W (Eds). Linnaeus Tercentenary: Progress in Invertebrate Taxonomy. Zootaxa 16:681-766. https://doi.org/10.11646/zootaxa.1668.1.30

Le Croy KA, Shew HW, Van Zandt PA (2013). Pollen presence on nocturnal moths in the Ketona Dolomite glades of Bibb County, Alabama. Southern Lepidopterists' News 35:136-142.

Mandal DK, Maulik DR (1991). Insecta: Lepidoptera: Heterocera: Noctuidae, Sphingidae and Geometridae pp 209-234. In: Fauna of Orissa (Part 3), State Fauna Series - 1, published by the Director, Zoological Survey of India, Kolkata.

Rath PC, Bose L, Subudhi H, Lenka S, Jambhulkar N (2020). Biodiversity of Pests of Rice in Odisha. International Journal of Current Microbiology and Applied Sciences 9(3):566-569. https://doi.org/10.20546/ijcmas.2020.903.066

Saha S, Raychaudhuri D (1998). Moths of Buxa Tiger Reserve, Jalpaiguri, West Bengal. Zoos' Print pp 24.

Sanyal AK, Alfred JRB, Venkataraman K, Tiwari SK, Mitra S (2012). Status of Biodiversity of West Bengal. Zoological Survey of India, Kolkata.

Sharma AK, Bisen UK (2013). Taxonomic documentation of insect pest fauna of vegetable ecosystem collected in light trap. International Journal of Environmental Science: Development and Monitoring 4(3):1-8.

Shubhalaxmi V, Kendrick RC, Vaidya A, Kalagi N, Bhagwat A (2011). Inventory of moth fauna (Lepidoptera: Heterocera) of the northern Western Ghats, Maharashtra, India. Journal of the Bombay Natural History Society 108(3):183-205.

Singh N, Ahmad J, Joshi R (2018). Moths (Lepidoptera) diversity of district Koderma, Jharkhand. Journal of Entomology and Zoology Studies 6(2):1253-1263.

Singh N, Ranjan R (2016). Additions to the moth fauna of Dalma Wildlife Sanctuary, Jharkhand (India). Records of Zoological Survey of India 116(4):323-336.

Soggard J (2009). Moths and caterpillars of the North Woods. Kollath-Stensaas Publishing, Duluth.

Sondhi S, Sondhi Y, Roy P, Kunte K (2021). Moths of India. v. 2.52. Indian Foundation for Butterflies. Retrieved 2021 February 2 from http://www.mothsofindia.org/

Sridhar V, Srinivas P (2019). Report of South American tomato moth, Tuta absoluta (Meyrick) from Odisha. Pest Management in Horticultural Ecosystems 25(1):119-120.

Tripathy MK, Rout M, Tripathy A (2018). Population dynamics of teak defoliator, Hyblaea puera Cramer at coastal Odisha, India. Journal of Entomology and Zoology Studies 6(5):2378-2387.

Van Nieukerken EJ, Kaila L, Kitching IJ, Kristensen NP, Lees DC, Minet J, ... Zwick A (2011). Order Lepidoptera. In: Zhang ZQ (Ed). Animal Biodiversity: An outline of higher-level classification and survey of taxonomic richness. Zootaxa 3148:212-221. https://doi.org/10.11646/zootaxa.3148.1.41

Vattakaven T, George R, Balasubramanian D, Réjou-Méchain M, Muthusankar G, Ramesh B, Prabhakar R (2016). India Biodiversity Portal: An integrated, interactive and participatory biodiversity informatics platform. Retrieved 2021 February 2 from https://indiabiodiversity.org/

Zahiri R, Kitching IJ, Lafontaine JD, Mutanen M, Kaila L, Holloway JD, Wahlberg N (2010). A new molecular phylogeny offers hope for a stable family level classification of the Noctuoidea (Lepidoptera). Zoologica Scripta 40(2):158173. http://www.dx.doi.org/10.1111/j.1463-6409.2010.00459.x

Zahiri R, Holloway JD, Kitching IJ, Lafontaine D, Mutanen M, Wahlberg N (2011). Molecular phylogenetics of Erebidae (Lepidoptera, Noctuoidea). Systematic Entomology 37(1):102-124. https://doi.org/10.1111/j.13653113.2011.00607.x 
Pattanaik N et al. (2021). Not Sci Biol 13(3):10934

OPEN ACCESS

(c) (i)

The journal offers free, immediate, and unrestricted access to peer-reviewed research and scholarly work. Users are allowed to read, download, copy, distribute, print, search, or link to the full texts of the articles, or use them for any other lawful purpose, without asking prior permission from the publisher or the author.

License - Articles published in Notulae Scientia Biologicae are Open-Access, distributed under the terms and conditions of the Creative Commons Attribution (CC BY 4.0) License.

(C) Articles by the authors; SHST, Cluj-Napoca, Romania. The journal allows the author(s) to hold the copyright/to retain publishing rights without restriction. 